\title{
Two Species and Three Species Ecological Modeling - Homotopy Analysis
}

\author{
Venkata Sundaranand Putcha* \\ Center for Mathematical Sciences - DST, C.R. Rao Advanced Institute of Mathematics, \\ Statistics and Computer Science (AIMSCS), UoH Campus, \\ Gachibowli, Hyderabad, A.P \\ India
}

\section{Introduction}

It is observed that big trees are older than little trees and there are more little trees than big trees since not every little tree grows up to be a big tree - most die young. But seeds produced and shed by the bigger trees will naturally form little trees. It can also be noticed that there are some dead needles and leaves on the ground and some standing dead trees which will eventually fall to the soil, the result of the deaths of those young trees and plant parts. It is natural that the live trees have roots in the soil formed partly from those dead leaves and logs and surmise that the trees obtain some nutrients from them Mangroves are the forests positioned at the convergence of land and Sea in inter tidal zones of the world. Mangrove forests are architecturally simple when compared to rainforests, often lacking under storey of leafs and shrubberies and are generally less species rich than other tropical forests. Mangroves have been heavily used traditionally for food, timber, fuel and medicine, and presently occupy about $181000 \mathrm{~km}^{2}$ of tropical and subtropical coastline. Mangroves are a precious ecological and economic resource, being vital nursery grounds and breeding sites for birds, fish, crustaceans, shellfish, reptiles,Polychaete, Crabs, Prawn, Zooplankton and mammals; a renewable source of wood; accrual sites for sediment, contaminants, carbon and nutrients; and offer Fortification against coastal erosion. Major reasons for obliteration of mangroves are urban development, aquaculture, mining and over exploitation for timber, fish, crustaceans and shellfish.

Ecological modeling does not deal directly with natural objects, it deals with the mathematical objects and operations which are offered as analogs of nature and natural processes. These mathematical models do not contain all information about nature that we may know, but only what we think are the most pertinent for the problem at hand. Ecological modeling helps us understand the logic of our thinking about nature to help us avoid making plausible arguments that may not be true or only true under certain restrictions. It helps us avoid wishful thinking about how we would like nature to be in favor of rigorous thinking about how nature might actually work.

It has been observed by many ecologist that Living organisms and their non living environment are inseparable, interrelated and interacts up on each other.When species interact the population dynamics of each species is affected. We consider here systems involving

*Dedicated to my grandmother Balatripura Sundari Putcha 
two-species systems and three-species systems. There are three main types of interaction, (i) If the growth rate of one population is decreased and the other increased the populations are in a predator-prey situation, (ii) If the growth rate of each population is decreased then it is competition, (iii) If each population's growth rate is enhanced then it is called mutualism or symbiosis.

Eco system comprising organisms and a biotic environment is a functional unit of ecology and non linear differential equations of first order are used to represent complex ecological phenomena. In 1925 Volterra [20] first proposed a simple model for the predation of one species by another to explain the oscillatory levels of certain fish catches in the Adriatic with the following assumptions (i) The prey in the absence of any predation grows unboundedly in a Malthusian way (ii) The effect of the predation is to reduce the prey's per capita growth rate by a term proportional to the prey and predator populations (iii) In the absence of any prey for sustenance the predator's death rate results in exponential decay (iv) The prey's contribution to the predators' growth rate is proportional to the available prey as well as to the size of the predator population. Ayala etal [21] studied the theoretical models through experimental tests. Later Albrecht etal [22], Gopalaswamy [23], Brown etal [24] studied the stability of two species systems.

The systems of non linear differential equations are perfect and an abstract representation of the real problem and attracted the attention of mathematicians [3], [4], [7] and [9] and ecologists. May [25] studied the nonlinear aspects of competition between three species. Schuster etal [26] and Zhang Jinyan [27] studied three species systems.So it is of great interest to formulate a mathematical models on Two Species and Three Species Ecological systems and find their approximate analytical solutions. In general construction of analytic approximations of nonlinear problems with strong nonlinearity is not easy and constructed Solutions are mainly determined by the type of nonlinearity and the corresponding technique. The region of convergence of obtained solutions depend on physical parameters rather than the analytical technique.

Though numerical techniques can be used to solve the nonlinear system of differential equations the functional form of reasonably good solution, which is of vital use for detailed study of the system, can be constructed by Homotopy methods. In general it is difficult to obtain analytic approximations of nonlinear problems with strong nonlinearity. Traditionally, solution expressions of a nonlinear problem are mainly determined by the type of nonlinear equations and the employed analytic techniques, and the convergence regions of solution series are strongly dependent of physical parameters.

The Homotopy analysis method HAM, initially proposed by Liao in his Ph.D. thesis [15], is a powerful analytic method for nonlinear problems. A systematic and clear exposition on HAM is given in [16]. In recent years, this method has been successfully employed by many authors Sami etal [32], Francisco M. Fernandez [33], Faghidian [34], Shijun Liao [35], Zoua L [36], Rafei M [37], Cheng-shi Liu etal [38], Vipul K. Baranwal etal [39] to solve many types of nonlinear problems in science and engineering.

The references cited may not be exhaustive since much literature is available on methods for solving nonlinear systems by approximate analytical solutions in general and Homotopy methods in particular. This chapter is organized as follows. In section 2, outline of Perturbation method, Adomian decomposition method for system, Homotopy perturbation method, Homotopy analysis method and Homotopy analysis method for system to find approximate analytical solutions of non linear differential equations is presented. Section 
3 is concerned with the two species systems and present approximate analytical solutions obtained by using perturbation method, Homotopy perturbation method and Homotopy analysis method. In section 4, Three species ecological systems are considered and are solved by using perturbation method, Adomian decomposition method and Homotopy analysis method. While remembering the famous words "In learning Science examples are useful than rules" of Isaac Newton numerical examples are presented to illustrate the simplicity and accuracy of the methods in section 5. Section 6 presents the summary and conclusions.

\section{Methods}

\subsection{Perturbation method}

Perturbation method is a widely used method to solve non linear differential equations.Consider a system of nonlinear differential equations of the type $\dot{x}_{1}(t)=$ $f_{1}\left(x_{1}, x_{2}, x_{3}, t\right)+\varepsilon g_{1}\left(x_{1}, x_{2}, x_{3}, t\right), \dot{x}_{2}(t)=f_{2}\left(x_{1}, x_{2}, x_{3}, t\right)+\varepsilon g_{2}\left(x_{1}, x_{2}, x_{3}, t\right), \dot{x}_{3}(t)=$ $f_{3}\left(x_{1}, x_{2}, x_{3}, t\right)+\varepsilon g_{3}\left(x_{1}, x_{2}, x_{3}, t\right)$ where the functions $f_{1}, f_{2}, f_{3}$ are linear and the functions $g_{1}, g_{2}, g_{3}$ are nonlinear and $\varepsilon$ is a small parameter, satisfying the initial conditions $x_{1}(0)=$ $a, x_{2}(0)=b, x_{3}(0)=c$. The solutions $x_{1}(t), x_{2}(t), x_{3}(t)$ are expressed as a power series of $\varepsilon$ as $x_{1}(t)=x_{1,0}(t)+\varepsilon x_{1,1}(t)+\varepsilon^{2} x_{1,2}(t)+\ldots, x_{2}(t)=x_{2,0}(t)+\varepsilon x_{2,1}(t)+\varepsilon^{2} x_{2,2}(t)+\ldots, x_{3}(t)=$ $x_{3,0}(t)+\varepsilon x_{3,1}(t)+\varepsilon^{2} x_{3,2}(t)+\ldots$. . These series will converge rapidly if $\varepsilon$ is very small. The terms $x_{1,0}(t), x_{2,0}(t), x_{3,0}(t)$ are the solutions of the corresponding linear equations $\dot{x}_{1}(t)=f_{1}\left(x_{1}, x_{2}, x_{3}, t\right), \dot{x}_{2}(t)=f_{2}\left(x_{1}, x_{2}, x_{3}, t\right), \dot{x}_{3}(t)=f_{3}\left(x_{1}, x_{2}, x_{3}, t\right)$ and are known as the generating solutions. The terms $x_{1,1}(t), x_{2,1}(t), x_{3,1}(t), x_{1,2}(t), x_{2,2}(t), x_{3,2}(t)$ are called correction terms. It is an established fact that analytic approximations of nonlinear problems often fail for strong nonlinearities and the perturbation approximations work only for weak nonlinearities.

\subsection{Adomian decomposition method for system of differential equations}

The decomposition method was developed by G. Adomian [5] to linear and non-linear differential and partial differential equations. With the assumptions like weak non-linearity and small perturbation, the solution of the simpler mathematical problem may not be a good approximation to the solution of the original problem. The advantage of the decomposition method is that it provides analytical approximation to a wide class of non-linear equations without linearization as in perturbation, closure approximation or discritization methods. In this section, we describe the Adomain's decomposition method for non-linear matrix differential equation. Consider a matrix differential equation $F X(t)=G(t)$, where $F$ represents a general non-linear ordinary differential operator involving both linear and non-linear terms, $X(t)$ and $G(t)$ are square matrices. Decompose the nonlinear operator into $M+N+R$, where $M$ is easily invertible operator and $R$ is the remainder of the linear operator and $N$ represents non-linear operator. Thus the equation

$$
F X(t)=G(t)
$$

may be written as

$$
\begin{aligned}
& M X(t)+R X(t)+N X(t)=G(t) \\
& \text { i.e., } \quad M X=G-R X-N X \\
& \text { i.e., } M^{-1} M X=M^{-1} G-M^{-1} R X-M^{-1} N X
\end{aligned}
$$


since $M$ is easily invertible operator. If this corresponds to an initial value problem, the integral operator $M^{-1}$ may be a definite integral from $t_{0}$ to $t$. Solving (3) for $X$ yields,

$$
X=E_{1}+E_{2} t+M^{-1} G-M^{-1} R X-M^{-1} N X
$$

The non linear term $N X$ will be equated to $\sum_{n=0}^{\infty} A_{n}$, where the $A_{n}$ 's are special polynomials, which can be defined further by (7), and $X$ will be decomposed into $\sum_{n=0}^{\infty} X_{n}$, with $X_{0}$ the initial approximate matrix identified as $E_{1}+E_{2}+M^{-1} G$. Thus (4) can be expressed as

$$
\sum_{n=0}^{\infty} X_{n}=X_{0}-M^{-1} R \sum_{n=0}^{\infty} X_{n}-M^{-1} \sum_{n=0}^{\infty} A_{n}
$$

Consequently we can write, the matrix approximations,

$$
X_{1}=-M^{-1} R X_{0}-M^{-1} A_{0}, X_{2}=-M^{-1} R X_{1}-M^{-1} A_{1}, \ldots, X_{n+1}=-M^{-1} R X_{n}-M^{-1} A_{n}(6)
$$

The matrix polynomials $A_{n}$ are generated for each non-linearity so that $A_{0}$ depends only on $X_{0}, A_{1}$ depends only on $X_{o}$ and $X_{1}, A_{2}$ depends on $X_{0}, X_{1}$ and $X_{2}$ and so on. All of the matrix components $X_{n}$ are calculable, and thus $X=\sum_{n=0}^{\infty} X_{n}$. If the series converges, then the $n^{\text {th }}$ partial sum $\Phi_{n}=\sum_{i=0}^{n-1} X_{i}$ will be the solution since $\Phi_{n} \longrightarrow \sum_{i=0}^{n-1} X_{i}=X$. To calculate the matrix polynomials $A_{n}$ 's consider an equation for which $\mathrm{X}(\mathrm{t})$ is the solution, containing a non-linear term $N X=Q(X)=\sum_{n=0}^{\infty} A_{n}$. These $A_{n}$ matrix polynomials are defined as

$$
\begin{aligned}
& A_{0}=Q\left(X_{0}\right), A_{1}=\left(e^{X_{1} d / d x_{0}}-1\right) Q\left(X_{0}\right) \\
& A_{2}=\left(e^{X_{2} d / d x_{0}}-1\right) Q\left(X_{0}\right)+\left(e^{X_{1} d / d x_{0}}-1\right)\left(e^{X_{2} d / d x_{0}}-1\right) Q\left(X_{0}\right)
\end{aligned}
$$

Thus $\sum_{i=0}^{n} A_{i}=Q\left(X_{0}\right)+\left(X-X_{0}\right) d Q / d X_{0}+\ldots$. That is, the partial sums consist of the essential terms of a Taylor expansion about the function $X_{0}(t)$ rather than about a point. Thus, addition of the first $(\mathrm{n}+1)$ terms of the $A_{0}, A_{1}, \ldots, A_{n}$ approaches

$\left(e^{X d / d x_{0}}-1\right) Q\left(X_{0}\right)$. With the product terms, products of $\mathrm{n}$ ! occur in the denominator which also can be ignored after some $\mathrm{n}$. Thus $A_{n}$ can be written as

$$
A_{n}=B_{n} Q\left(X_{0}\right), \text { where } B_{n}=C_{n} \sum_{j=0}^{n} B_{j}, n \geq 1, B_{0}=1, C_{n}=\left(e^{X_{n} d / d x_{0}}-1\right)
$$

\subsection{Homotopy perturbation method}

Various perturbation methods applied to solve nonlinear problems are based on the strict assumption of the existence of a small parameter which is not satisfied in many non linear problems. To overcome this difficulty in 1999, J H He [14] proposed a method called Homotopy perturbation method which is a combination of the classical perturbation technique and Homotopy technique. To explain the basic idea of the Homotopy perturbation method for solving nonlinear differential equations, we consider the following nonlinear differential equation

$$
A(u)-f(r)=0, r \in \Omega
$$

satisfying the boundary condition

$$
B\left(u, \frac{\partial u}{\partial t}\right)=0, r \in \Gamma
$$


where $A$ is a general differential operator, B is a boundary operator, $f(r)$ is a known analytical function, $\Gamma$ is the boundary of domain $\Omega$ and $\frac{\partial}{\partial t}$ denotes differentiation along the normal drawn outwards from $\Omega$. The operator $A$ can be divided into a linear part $\mathrm{L}$ and a nonlinear part $\mathrm{N}$ as

$$
L(v)+N(v)-f(r)=0
$$

Construct a Homotopy $v(r, p): \Omega \times[0,1] \longrightarrow \mathbb{R}$ where the embedding parameter $p \in$ $[0,1], r \in \Omega$, the initial guess $u_{0}$ satisfying

$$
H(v, p)=(1-p)\left[L(v)-L\left(u_{0}\right)\right]+p[A(v)-f(r)]=0,
$$

The above equation is equivalent to $H(v, p)=L(v)-L\left(u_{0}\right)+p L\left(u_{0}\right)+p[N(v)-f(r)]=0$. As $p$ changes from 0 to 1 the initial guess $u_{0}(r)$ of $v(r, p)$ goes to $u(r)$. In this context $L(u)-L\left(u_{0}\right)$ and $A(v)-f(r)$ are homotopic in the topological sense. Now the solution of (12) is of the form $v=v_{0}+p v_{1}+p^{2} v_{2}+\ldots$. The approximate analytical solution of (9) is given by $u=\lim _{p \longrightarrow 1} v=\sum_{i=0}^{\infty} v_{i}$. This series is convergent for most cases, however, the convergent rate depends upon the nonlinear operator $A(v)$ and 1. From [12] the series converges if (i) the second derivative of $N(v)$ with respect to $\mathrm{v}$ must be small, because the parameter $\mathrm{p}$ may be relatively large, (i.e. $p \longrightarrow 1$ ) and (ii) the norm of $L^{-1} \frac{\partial N}{\partial v}$ must be smaller than one. perturbation techniques use perturbation quantities to transfer a nonlinear problem into an infinite number of linear sub-problems and then approximate it by the sum of solutions of the first several sub-problems. The existence of perturbation quantities is obviously a cornerstone of perturbation techniques, however, it is the perturbation quantity that brings perturbation techniques some serious restrictions.It is not necessary for every nonlinear problem to contain such a perturbation quantity. This is a major restriction of perturbation method.

\subsection{Homotopy analysis method}

From the literature it is observed that if a nonlinear problem has a unique solution, there may exist an infinite number of different solution expressions whose convergence region and rate are dependent on an auxiliary parameter. Homotopy analysis method was first proposed by Liao [15] based on Homotopy, a fundamental concept in topology and differential geometry. The HAM is based on construction of Homotopy which continuously deforms an initial guess approximation to the exact solution of the given problem. An auxiliary linear operator is chosen to construct the Homotopy and an auxiliary linear parameter is used to control and adjust the region of the convergence region and rate of the solution series, which is not possible in the other methods like perturbation techniques, Homotopy perturbation methods, decomposition methods. The Homotopy analysis method provides the greater flexibility in choosing initial approximations and auxiliary linear operators. Moreover, unlike all previous analytic techniques, the Homotopy analysis method provides great freedom to use different base functions to express solutions of a nonlinear problem so that one can approximate a nonlinear problem more efficiently by means of better base functions. Thus, this method is valid for nonlinear problems with strong nonlinearity. Furthermore, the Homotopy analysis method logically contains some previous techniques such as perturbation method, AdomianŠs decomposition method, LyapunovŠs artificial small parameter method, and the $\delta$-expansion method. Thus, it can be regarded as a unified or generalized theory of these previous methods. Consider the following non linear problem

$$
N(u(x, t))=0, t>0
$$


where $N$ is a nonlinear operator and $u(x, t)$ is unknown function of the independent variables $x, t$. The zero order deformation equation

$$
(1-q) L\left[\phi(x, t, q)-u_{0}(x, t)\right]=q h H(x, t) N(\phi(x, t, q))
$$

where $q \in[0.1]$ is the Homotopy or embedding parameter, $h \neq 0$ an auxiliary parameter, $H(x, t) \neq 0$ is an auxiliary function, $\mathrm{L}$ is an auxiliary linear operator, $u_{0}(x, t)$ an initial guess of $u(x, t)$ and $\phi(x, t, q)$ is an unknown function. By substituting $q=0$ and $q=1$ in (14) it can easily be observed that $\phi(x, t, q)$ deforms continuously from the initial guess $u_{0}(x, t)$ to the exact solution $u(x, t)$ as the embedding parameter $\mathrm{q}$ increases from 0 to 1 . By expanding $\phi(x, t, q)$ in a Taylor series, we get

$$
\begin{aligned}
\phi(x, t, q) & =u_{0}(x, t)+\sum_{m=1}^{\infty} u_{m}(x, t) q^{m} \\
\text { where } u_{m}(x, t) & =\frac{1}{m !} \frac{\partial^{m} \phi(x, t, q)}{\partial q^{m}}
\end{aligned}
$$

The convergence of the series (15) is controlled by $h$. Assume that the auxiliary parameter $h$ the auxiliary function $H$, the initial approximation $u_{0}(x, t)$ and the auxiliary linear operator $L$ are so properly chosen that the series (15) converges at $q=1$. Then, exact solution of (15) is given by

$$
u(x, t)=u_{0}(x, t)+\sum_{m=1}^{\infty} u_{m}(x, t) q^{m}
$$

Now the functions $u_{m}(x, t)$ for $m=1,2,3, \ldots$ are determined by differentiating the zero order deformation equation (14) $m$ times with respect to the embedding parameter $q$, dividing by $m$ ! and then setting $q=0$ we get the $m^{\text {th }}$ order deformation equation

$$
\begin{gathered}
L\left[u_{m}(x, t)-\chi_{m} u_{m-1}(x, t)=h H(t) R_{m}\left(u_{m-1}(x, t)\right)\right. \\
\text { where } \quad R_{m}\left(u_{m-1}(x, t)\right)=\frac{1}{(m-1) !} \frac{\partial^{m-1} \phi(x, t, q)}{\partial q^{m-1}} \\
\qquad u_{m}=\left\{u_{0}(x, t), u_{1}(x, t), u_{3}(x, t), \ldots, u_{m}(x, t)\right\} \\
\chi_{m}=\left\{\begin{array}{c}
0, m \leq 1 \\
1, \text { otherwise }
\end{array}\right.
\end{gathered}
$$

For any given operators $\mathrm{L}$ and $\mathrm{N}$ we get the $m^{\text {th }}$ order deformation Equation (18) and solving it we get $u_{m}(x, t)$ for different $m$. The solution of problem (13) is obtained by substituting the obtained $u_{m}(x, t) S$ s in (17) and choosing a suitable value of $h$ for the convergence of the series.

Homotopy analysis method is based on the following assumptions: (i) There exists the solution of the zeroth order deformation equation in the whole region of the embedding parameter $q \in[0,1]$. (ii) All the higher order deformation equations have solutions. (iii) All Taylor series expanded in the embedding parameter $q$ converge at $q=1$. Till now, there are no rigorous theories to direct us to choose the initial approximations, auxiliary linear operators, auxiliary functions, and auxiliary parameter are available in the literature though some fundamental rules based on practical problems such as the rule of solution expression, the rule of coefficient ergodicity, and the rule of solution existence, which play important roles within the Homotopy analysis method are available. 


\subsection{Homotopy analysis method for system of differential equations}

Consider the system of differential equations

$$
\left.N_{i}\left(x_{i}(t)\right)\right)=y_{i}(t), i=1,2,3, \ldots, n
$$

where $N_{i}$ are nonlinear operators, $t$ denotes the independent variable, $x_{i}(t)$ are unknown functions, and $y_{i}(t)$ are known analytic functions representing the nonhomogeneous terms. Equation (22) becomes a homogeneous equation if $y_{i}(t)=0$. The zeroth-order deformation equation is constructed as

$$
(1-q) L\left[\phi_{i}(t, q)-x_{i, 0}(t)\right]=q h\left\{N_{i}\left(\phi_{i}(t, q)\right)-y_{i}(t)\right\}
$$

where $q \in[0,1]$ is an embedding parameter, $h$ is a nonzero auxiliary function, $L$ is an auxiliary linear operator, $x_{i, 0}(t)$ are the initial guesses of $y_{i}(t)$, and $\phi_{i}(t, q)$ are unknown functions. It is important to note that one has great freedom to choose the auxiliary objects such as $h$ and $L$ in HAM. Obviously, when $q=0$ and $q=1$, both

$$
\phi_{i}(t, 0)=x_{i, 0}(t) ; \phi_{i}(t, 1)=x_{i}(t)
$$

hold. Thus, as $q$ increases from 0 to 1 , the solutions $\phi_{i}(t, q)$ vary from the initial guesses $x_{i, 0}(t)$ to the solutions $x_{i}(t)$. By expanding $\phi_{i}(t, q)$ in Taylor series with respect to $q$, we get

$$
\begin{gathered}
\phi_{i}(t, q)=x_{i, 0}(t)+\sum_{m=1}^{\infty} x_{i, m}(t) q^{m} \\
\text { where } \quad x_{i, m}(t)=\left.\frac{1}{m !} \frac{\partial^{m} \phi_{i}(t, q)}{\partial q^{m}}\right|_{q=0}
\end{gathered}
$$

If the auxiliary linear operator $L$, the initial guesses $x_{i, 0}(t)$, the auxiliary parameters $h$, and the auxiliary functions are chosen properly such that the series (25)converges at $q=1$ and

$$
\phi_{i}(t, 1)=x_{i}(t)=x_{i, 0}(t)+\sum_{m=1}^{\infty} x_{i, m}(t)
$$

will be one of the solution of the original nonlinear equation. For the choice of $h=-1$, (23) becomes

$$
(1-q) L\left[\phi_{i}(t, q)-x_{i, 0}(t)\right]+q h\left\{N_{i}\left(\phi_{i}(t, q)\right)-y_{i}(t)\right\}=0
$$

Define the vector

$$
\vec{x}_{i, n}(t)=\left[x_{i, 0}(t), x_{i, 1}(t), \ldots, x_{i, n}(t)\right]
$$

The $m^{\text {th }}$ order deformation equation is obtained by differentiating the equation (23) with respect to the embedding parameter $q, m$ times, then setting $q=0$, and finally dividing them by $m$ ! and is given by

$$
\begin{aligned}
& L\left[x_{(}(i, m)-\chi_{m} x_{i, m-1}(t)=h H(t) R_{i, m}\left(\vec{x}_{i, m-1}\right)\right. \\
& \text { where } R_{i, m}\left(\vec{x}_{i, m-1}\right)=\frac{1}{(m-1) !} \frac{\partial^{m-1}\left\{N_{i}\left[\phi_{i}(t, q)\right]-y_{i}(t)\right\}}{\partial q^{m-1}} \mid q=0 \\
& \text { and } \\
& \qquad \chi_{m}=\left\{\begin{array}{c}
0, m \leq 1 \\
1, \text { otherwise }
\end{array}\right.
\end{aligned}
$$

It is evident that $x_{i, m-1}(t)(m \geq 1)$ is governed by the linear equation (30) with the linear initial or boundary conditions that come from the problem of consideration. 


\section{Two species ecological systems}

Consider a two species ecological system represented by Lokta Volterra equations of the form

$$
\begin{aligned}
& \dot{N}_{1}(t)=N_{1}(t)\left[a_{1}-b_{11} N_{1}(t)-b_{12} N_{2}(t)\right. \\
& \dot{N}_{2}(t)=N_{2}(t)\left[a_{2}-b_{21} N_{1}(t)-b_{22} N_{2}(t)\right.
\end{aligned}
$$

where $N_{1}(t), N_{2}(t)$ denote the densities of the two species, $a_{1}, b_{11}, a_{2}, b_{22}$ are the logistic parameters for the first and second species and $b_{12}, b_{21}$ arise from from the inhibiting effect on the growth of the first species due to the presence of the second and from the effect on the growth of the second species due to the presence of the first species. Assume that the per capita growth rate of each population at any instant is a linear function of the densities of the two competing populations at that instant. Each population would grow logistically in the absence of the other. In 1977 [17] obtained the exact solutions of the Lokta Volterra equations $\dot{n}_{1}(t)=n_{1}(t)\left[a_{1}-c_{1} n_{2}(t)\right], \dot{n}_{2}(t)=n_{2}(t)\left[-a_{2}-c_{2} n_{1}(t)\right]$ when $a_{1}=a_{2}$. The form of exact solution of this system when all the coefficients $a_{1}, a_{2}, c_{1}$ and $c_{2}$ time varying and $a_{1}(t)=a_{2}(t), c_{1}(t)=c_{2}(t)$ Wilson [18]. With another assumption $\left[a_{1}(t)-a_{2}(t)\right] c_{1}(t) c_{2}(t)=c_{2}(t) \dot{c}_{1}(t)-c_{1}(t) \dot{c_{2}}(t)$ Burnside [19] gave the exact solution. Murty and Rao [3] constructed approximate analytical solution of $\dot{n}_{1}(t)=n_{1}(t)\left[a_{1}-b_{11} n_{1}(t)-\right.$ $\left.b_{12} n_{2}(t)\right], \dot{n}_{2}(t)=n_{2}(t)\left[-a_{2}-b_{21} n_{1}(t)\right]$ with the assumption that the prey population $n_{1}(t)$ interfere with one another but not the predators $n_{2}(t)$. As all these assumptions may not depict the real world phenomena it is important to find the approximate analytical solutions of the system of equations (33-34) because of their non linear nature.

\subsection{Two species system - perturbation method}

The equilibrium point of (33) is given by

$$
\begin{aligned}
& N_{1}^{*}=\left[a_{1} b_{22}-a_{2} b_{12}\right] /\left[b_{11} b_{22}-b_{21} b_{12}\right] \\
& N_{2}^{*}=\left[a_{2} b_{11}-a_{1} b_{21}\right] /\left[b_{11} b_{22}-b_{21} b_{12}\right]
\end{aligned}
$$

Define new variables by

$$
\begin{aligned}
& X(t)=N_{1}(t)-N_{1}^{*} \\
& Y(t)=N_{2}(t)-N_{2}^{*}
\end{aligned}
$$

i.e., the variables $X$ and $Y$ represent densities of the two populations deviating from the equilibrium point. Now the equations (33) become

$$
\begin{aligned}
& \dot{X}(t)=-b_{11} N_{1}^{*} X(t)-b_{12} N_{1}^{*} Y(t)-b_{11} X^{2}(t)-b_{12} X(t) Y(t) \\
& \dot{Y}(t)=-b_{21} N_{2}^{*} X(t)-b_{22} N_{1}^{*} Y(t)-b_{22} Y^{2}(t)-b_{21} X(t) Y(t)
\end{aligned}
$$

By introducing the parameter $\mu$ in to non linear terms of the above equation we get

$$
\begin{aligned}
& \dot{X}(t)=-b_{11} N_{1}^{*} X(t)-b_{12} N_{1}^{*} Y(t)-\mu b_{11} X^{2}(t)-\mu b_{12} X(t) Y(t) \\
& \dot{Y}(t)=-b_{21} N_{2}^{*} X(t)-b_{22} N_{1}^{*} Y(t)-\mu b_{22} Y^{2}(t)-\mu b_{21} X(t) Y(t)
\end{aligned}
$$


We seek solutions of the (33) in the form

$$
\begin{gathered}
X(t)=X_{0}+\mu X_{1}(t)+\mu^{2} X_{2}(t)+\ldots \\
Y(t)=Y_{0}+\mu Y_{1}(t)+\mu^{2} Y_{2}(t)+\ldots
\end{gathered}
$$

By equating like powers of $\mu$ after substituting (41),(42),(43),(44) in (37), (38),(39), (40) we get

$$
\begin{aligned}
& \dot{X}_{0}(t)=-b_{11} N_{1}^{*} X_{0}(t)-b_{12} N_{1}^{*} Y_{0}(t) \\
& \dot{Y}_{0}(t)=-b_{21} N_{2}^{*} X_{0}(t)-b_{22} N_{1}^{*} Y_{0}(t) \\
& \dot{X}_{1}(t)=-b_{11} N_{1}^{*} X_{1}(t)-b_{12} N_{1}^{*} Y_{1}(t)-b_{11} X_{0}^{2}(t)-b_{12} X_{0}(t) Y_{0}(t) \\
& \dot{Y}_{1}(t)=-b_{21} N_{2}^{*} X_{1}(t)-b_{22} N_{1}^{*} Y_{1}(t)-b_{22} Y_{0}^{2}(t)-b_{21} X_{0}(t) Y_{0}(t)
\end{aligned}
$$

By applying the initial conditions $X(0)=p_{0}, Y(0)=q_{0}$, zero initial conditions for correction terms and by using Laplace transforms the solutions of the above equations are given by

$$
\begin{aligned}
& X_{0}(t)=\frac{1}{\alpha-\beta}\left[p_{0}\left(\alpha e^{\alpha t}-\beta e^{\beta t}\right)+A_{1}\left(e^{\alpha t}-e^{\beta t}\right)\right] \\
& Y_{0}(t)=\frac{1}{\alpha-\beta}\left[q_{0}\left(\alpha e^{\alpha t}-\beta e^{\beta t}\right)+A_{2}\left(e^{\alpha t}-e^{\beta t}\right)\right]
\end{aligned}
$$

where $A_{1}=b_{22} N_{2}^{*} p_{0}-b_{12} N_{1}^{*} q_{0}, A_{2}=b_{11} N_{1}^{*} q_{0}-b_{21} N_{2}^{*} p_{0}, \alpha=-B_{1}+\sqrt{B_{1}^{2}-4 C_{1}} / 2$,

$$
\beta=-B_{1}-\sqrt{B_{1}^{2}-4 C_{1}} / 2, B_{1}=\left(b_{11} N_{1}^{*}+b_{22} N_{2}^{*}, C_{1}=\left(b_{11} b_{22}-b_{21} b_{12}\right) N_{1}^{*} N_{2}^{*}\right.
$$

Now $X_{1}(t)$ and $X_{2}(t)$ are given by

$$
\begin{gathered}
X_{1}(t)=P_{1} \alpha e^{\alpha t}+P_{2} e^{\beta t}+P_{3} e^{2 \alpha t}+P_{4} e^{2 \beta t}+P_{5} e^{(\alpha+\beta) t} \\
Y_{1}(t)=Q_{1} \alpha e^{\alpha t}+Q_{2} e^{\beta t}+Q_{3} e^{2 \alpha t}+Q_{4} e^{2 \beta t}+Q_{5} e^{(\alpha+\beta) t} \\
\text { where the constants } P_{i}, Q_{i}(i=1,2,3,4,5) \text { are given by } \\
P_{1}=\frac{D_{1} \alpha+L_{1}}{\alpha(\beta-\alpha)}+\frac{D_{2} \alpha+M_{1}}{(\alpha-\beta)(\alpha-2 \beta)}+\frac{D_{3} \alpha+N_{1}}{\beta(\beta-\alpha)} \\
P_{2}=\frac{D_{1} \beta+L_{1}}{(\beta-\alpha)(\beta-2 \alpha)}+\frac{D_{2} \beta+M_{1}}{\beta(\alpha-\beta)}+\frac{D_{3} \beta+N_{1}}{\alpha(\alpha-\beta)}, P_{3}=\frac{2 D_{1} \alpha+L_{1}}{\alpha(2 \alpha-\beta)}, P_{4}=\frac{2 D_{2} \beta+M_{1}}{\beta(2 \beta-\alpha)}, \\
P_{5}=\frac{D_{3}(\alpha+\beta)+N_{1}}{\alpha \beta}, \quad Q_{1}=\frac{E_{1} \alpha+L_{2}}{\alpha(\beta-\alpha)}+\frac{E_{2} \alpha+M_{2}}{(\alpha-\beta)(\alpha-2 \beta)}+\frac{E_{3} \alpha+N_{2}}{\beta(\beta-\alpha)} \\
Q_{2}=\frac{E_{1} \beta+L_{2}}{(\beta-\alpha)(\beta-2 \alpha)}+\frac{E_{2} \beta+M_{2}}{\beta(\alpha-\beta)}+\frac{E_{3} \beta+N_{2}}{\alpha(\alpha-\beta)}, Q_{3}=\frac{2 E_{1} \alpha+L_{2}}{\alpha(2 \alpha-\beta)}, Q_{4}=\frac{2 E_{2} \beta+M_{2}}{\beta(2 \beta-\alpha)}, \\
Q_{5}=\frac{E_{3}(\alpha+\beta)+N_{2}}{\alpha \beta} \quad \text { and } L_{1}=b_{22} N_{2}^{*} D_{1}-b_{12} N_{1}^{*} E_{1}, \quad M_{1}=b_{22} N_{2}^{*} D_{2}-b_{12} N_{1}^{*} E_{2}
\end{gathered}
$$




$$
\begin{aligned}
& N_{1}=b_{22} N_{2}^{*} D_{3}-b_{12} N_{1}^{*} E_{3}, \quad L_{2}=b_{11} N_{1}^{*} E_{1}-b_{21} N_{2}^{*} D_{1}, M_{2}=b_{11} N_{1}^{*} E_{2}-b_{21} N_{2}^{*} D_{2}, \\
& N_{2}=b_{11} N_{1}^{*} E_{3}-b_{21} N_{2}^{*} D_{3} \text { where } D_{1}=-b_{11} B_{2}^{2}-b_{12} B_{2} C_{2}, \quad D_{2}=-b_{11} B_{3}^{2}-b_{12} B_{3} C_{3} \\
& D_{3}=2 b_{11} B_{2} B_{3}+b_{12}\left(B_{2} C_{3}+B_{3} C_{2}\right), \quad E_{1}=-b_{22} C_{2}^{2}-b_{12} B_{2} C_{2}, \quad E_{2}=-b_{22} C_{3}^{2}-b_{21} B_{3} C_{3}, \\
& E_{3}=2 b_{22} C_{2} C_{3}+b_{21}\left(B_{2} C_{3}+B_{3} C_{2}\right) \\
& B_{2}=\frac{\alpha p_{0}+A_{1}}{\alpha-\beta}, B_{3}=\frac{\beta p_{0}+A_{1}}{\alpha-\beta}, C_{2}=\frac{\alpha q_{0}+A_{2}}{\alpha-\beta}, C_{3}=\frac{\beta q_{0}+A_{2}}{\alpha-\beta}
\end{aligned}
$$

Now the second order approximation is given by $X(t) \simeq X_{0}(t)+X_{1}(t), Y(t) \simeq Y_{0}(t)+Y_{1}(t)$. Now the approximate analytical solution in terms of $N_{1}$ and $N_{2}$ is given by

$$
\begin{aligned}
N_{1}(t) \simeq & \frac{a_{1} b_{22}-a_{2} b_{12}}{b_{11} b_{22}-b_{21} b_{12}}+\left[P_{1}+\frac{p_{0} \alpha+A_{1}}{(\alpha-\beta)}\right] e^{\alpha t}+\left[P_{2}-\frac{p_{0} \beta+A_{1}}{(\alpha-\beta)}\right] e^{\beta t} \\
& +P_{3} e^{2 \alpha t}+P_{4} e^{2 \beta t}+P_{5} e^{(\alpha+\beta) t} \\
N_{2}(t) \simeq & \frac{a_{2} b_{11}-a_{1} b_{21}}{b_{11} b_{22}-b_{21} b_{12}}+\left[Q_{1}+\frac{q_{0} \alpha+A_{2}}{(\alpha+\beta)}\right] e^{\alpha t}+\left[Q_{2}-\frac{q_{0} \beta+A_{2}}{(\alpha-\beta)}\right] e^{\beta t} \\
& +Q_{3} e^{2 \alpha t}+Q_{4} e^{2 \beta t}+Q_{5} e^{(\alpha+\beta) t}
\end{aligned}
$$

The accuracy of the solution can be improved by finding higher order approximations.

\subsection{Two species system - Homotopy perturbation method}

Now we consider Lokta Volterra system (33) with the conditions that $b_{11}=0$ and $b_{22}=0$

$$
\begin{aligned}
& \dot{N}_{1}(t)=N_{1}(t)\left[a_{1}-b_{12} N_{2}(t)\right. \\
& \dot{N}_{2}(t)=N_{2}(t)\left[a_{2}-b_{21} N_{1}(t)\right]
\end{aligned}
$$

and apply the Homotopy Perturbation Method to construct a Homotopy of the above resultant system (53),(54) as follows

$$
\begin{aligned}
& (1-p)\left(\dot{v}_{1}-\dot{N}_{1_{0}}\right)+p\left(\dot{v}_{1}-v_{1}(t)\left[a_{1}-b_{12} v_{2}(t)\right]\right)=0 \\
& (1-p)\left(\dot{v}_{2}-\dot{N}_{2_{0}}\right)+p\left(\dot{v}_{2}-v_{2}(t)\left[a_{2}-b_{21} v_{1}(t)\right]\right)=0
\end{aligned}
$$

(. denotes differentiation with respect $t$ ) the initial approximations are given by

$$
\begin{aligned}
& v_{1,0}(t)=N_{1_{0}}(t)=N_{1}(0) \\
& v_{2,0}(t)=N_{2_{0}}(t)=N_{2}(0)
\end{aligned}
$$

and

$$
\begin{aligned}
& v_{1}=v_{1,0}+p v_{1,1}+p^{2} v_{1,2}+p^{3} v_{1,3}++p^{4} v_{1,4}+\ldots \\
& v_{2}=v_{2,0}+p v_{2,1}+p^{2} v_{2,2}+p^{3} v_{2,3}++p^{4} v_{2,4}+\ldots
\end{aligned}
$$


where the functions $v_{i j}(i=1,2, j=1,2,3)$ are to be determined. By using equations (57),(58),(59),(60) in equations (55), (56) we get

$$
\begin{aligned}
& (1-p)\left[\left(\dot{v}_{1,0}+p \dot{v}_{1,1}+P^{2} \dot{v}_{1,2}+P^{3} \dot{v}_{1,3}+\ldots\right)-\dot{N}_{1}(0)\right]+p\left[\left(\dot{v}_{1,0}+p \dot{v}_{1,1}+P^{2} \dot{v}_{1,2}+P^{3} \dot{v}_{1,3}+\ldots\right)\right. \\
& -\left(v_{1,0}+p v_{1,1}+p^{2} v_{1,2}+p^{3} v_{1,3}+\ldots\right)\left(a_{1}-b_{12} v_{2,0}+p v_{2,1}+p^{2} v_{2,2}+p^{3} v_{2,3}+\ldots\right)=0 \\
& (1-p)\left[\left(\dot{v}_{2,0}+p \dot{v}_{2,1}+P^{2} \dot{v}_{2,2}+P^{3} \dot{v}_{2,3}+\ldots\right)-\dot{N}_{2}(0)\right]+p\left[\left(\dot{v}_{2,0}+p \dot{v}_{2,1}+P^{2} \dot{v}_{2,2}+P^{3} \dot{v}_{2,3}+\ldots\right)\right. \\
& -\left(v_{2,0}+p v_{2,1}+p^{2} v_{2,2}+p^{3} v_{2,3}+\ldots\right)\left(a_{2}-b_{21} v_{1,0}+p v_{1,1}+p^{2} v_{1,2}+p^{3} v_{1,3}+\ldots\right)=0 \\
& \text { i.e., }\left(\dot{v}_{1,1}-a_{1} N_{1_{0}}+b_{12} N_{1_{0}} N_{2_{0}}\right) p+\left(\dot{v}_{1,2}-a_{1} v_{1}+b_{12} N_{1_{0}} v_{2_{1}}+b_{12} N_{2_{0}} v_{1_{1}}\right) p^{2}+ \\
& \left(\dot{v}_{1,3}-a_{1} v_{1_{2}}+b_{12} N_{1_{0}} v_{2_{2}}+b_{12} N_{2_{0}} v_{1,2}+b_{12} v_{1,1} v_{2,1}\right) P^{3}=0 \\
& \left(\dot{v}_{2,1}-a_{2} N_{1_{0}}+b_{21} N_{1_{0}} N_{2_{0}}\right) p+\left(\dot{v}_{2,2}+a_{2} v_{2_{1}}-b_{21} N_{1_{0}} v_{2_{1}}-b_{21} N_{2_{0}} v_{1_{1}}\right) p^{2}+ \\
& \left(\dot{v}_{2,3}-N_{2_{0}} b_{21} v_{1,2}-b_{21} v_{1,1} v_{2,1}+v_{2,2}\left(a_{2}-b_{21} N_{1_{0}}\right)\right) p^{3}=0
\end{aligned}
$$

By considering the initial conditions $v_{i j}=0, i=1,2, j=1,2,3$

$$
\begin{aligned}
& \dot{v}_{1,1}-a_{1} N_{1_{0}}+b_{12} N_{1_{0}} N_{2_{0}}=0 \dot{v}_{1,2}-a_{1} v_{1_{1}}+b_{12} N_{1_{0}} v_{2_{1}}+b_{12} N_{2_{0}} v_{1_{1}}=0 \\
& \dot{v}_{2,3}-N_{2_{0}} b_{21} v_{1,2}-b_{21} v_{1,1} v_{2,1}+v_{2,2}\left(a_{2}-b_{21} N_{1_{0}}\right)=0 \\
& \dot{v}_{2,1}-a_{2} N_{1_{0}}+b_{21} N_{1_{0}} N_{2_{0}}=0, \dot{v}_{2,2}+a_{2} v_{2_{1}}-b_{21} N_{1_{0}} v_{2_{1}}-b_{21} N_{2_{0}} v_{1_{1}}=0 \\
& \dot{v}_{2,3}-N_{2_{0}} b_{21} v_{1,2}-b_{21} v_{1,1} v_{2,1}+v_{2,2}\left(a_{2}-b_{21} N_{1_{0}}\right)=0
\end{aligned}
$$

From the above equations we get

$$
\begin{aligned}
v_{1,1}= & a_{1} N_{1_{0}} t-b_{12} N_{1_{0}} N_{2_{0}} t \\
v_{1,2}= & \frac{1}{2} N_{1_{0}}\left[-2 a_{1} b_{12} N_{2_{0}}+a_{1}^{2}+b_{12} a_{2} N_{2_{0}}-b_{12} b_{21} N_{1_{0}} N_{2_{0}}+b_{12}^{2} N_{2_{0}}^{2}\right] t^{2} \\
v_{1,3}= & -\frac{1}{6} N_{1_{0}}\left[4 a_{1} b_{12} b_{21} N_{1_{0}} N_{2_{0}}-3 a_{1} b_{12}^{2} N_{2_{0}}^{2}-3 a_{1} b_{12} a_{2} N_{2_{0}}-a_{1}^{3}+\right. \\
& \left.b_{12} a_{2}^{2} N_{2_{0}}+b_{12}^{3} N_{2_{0}}^{3}-4 b_{12}^{2} b_{21} N_{1_{0}} N_{2_{0}}^{2}\right] t^{3}
\end{aligned}
$$

$v_{2,1}=-a_{2} N_{2_{0}} t-b_{21} N_{1_{0}} N_{2_{0}} t$

$$
\begin{aligned}
v_{2,2}= & \frac{1}{2} N_{2_{0}}\left[2 a_{2} b_{21} N_{1_{0}}-a_{2}^{2}-b_{21} a_{1} N_{1_{0}}+b_{12} b_{21} N_{1_{0}} N_{2_{0}}+b_{21}^{2} N_{1_{0}}^{2}\right] t^{2} \\
v_{2,3}= & \frac{1}{6} N_{2_{0}}\left[4 a_{2} b_{12} b_{21} N_{1_{0}} N_{2_{0}}-4 b_{12} b_{21}^{2} N_{1_{0}}^{2} N_{2_{0}}^{2}-3 a_{1} a_{2} b_{21} N_{1_{0}}-3 a_{1} b_{21}^{2} N_{1_{0}}-2 a_{1} b_{12} b_{21} N_{1_{0}} N_{2_{0}}\right. \\
& \left.+b_{12}^{2} b_{21} N_{1_{0}} N_{2_{0}}^{2}+a_{1}^{2} b_{21} N_{1_{0}}+3 a_{2}^{2} b_{21} N_{1_{0}}-3 a_{2} b_{21}^{2} N_{1_{0}}^{2}+b_{21}^{3} N_{1_{0}}^{3}-a_{2}^{3}\right] t^{3}
\end{aligned}
$$


Now the approximate analytical solution containing four terms by using Homotopy Perturbation method is given by

$$
\begin{aligned}
& N_{1}(t)=\lim _{p \longrightarrow 1} v_{1}(t)=v_{1,1}(t)+v_{1,2}(t)+v_{1,3}(t)+v_{1,4}(t) \\
& N_{2}(t)=\lim _{p \longrightarrow 1} v_{2}(t)=v_{2,1}(t)+v_{2,2}(t)+v_{2,3}(t)+v_{2,4}(t)
\end{aligned}
$$

The accuracy of the solution can be improved by considering more terms in the above solution expressions.

\subsection{Two species system - homotopy analysis method}

Consider the system of differential equations representing a two species ecological system (33),(34) satisfying the initial conditions

$$
N_{1}(0)=4, N_{2}(0)=10
$$

According to Homotopy Analysis Method, the initial approximations of (118) are satisfying the initial conditions

$$
N_{1,0}(0)=1, N_{2,0}(0)=2
$$

and the auxiliary linear operators $\mathcal{L}$ for $i=1,2$ are

$$
\mathcal{L}\left[\phi_{i}(t, q)\right]=\frac{\left.\partial \phi_{i}(t, q)\right)}{\partial t}, i=1,2, \text { with } \mathcal{L}\left(\left[C_{i}\right]=0 .\right.
$$

and the nonlinear operators

$$
\begin{aligned}
& \mathcal{N}_{1}\left[\phi_{i}(t, q)\right]=\frac{\left.\partial \phi_{1}(t, q)\right)}{\partial t}-\phi_{1}(t, q)\left[a_{1}-b_{11} \phi_{1}(t, q)-b_{12} \phi_{2}(t, q)\right]=g_{1}(t) \\
& \mathcal{N}_{2}\left[\phi_{i}(t, q)\right]=\frac{\left.\partial \phi_{2}(t, q)\right)}{\partial t}-\phi_{2}(t, q)\left[a_{2}-b_{21} \phi_{1}(t, q)-b_{22} \phi_{3}(t, q)\right]=g_{2}(t)
\end{aligned}
$$

The zeroth-order deformation equation is constructed as

$$
\begin{aligned}
(1-q) \mathcal{L}\left[\phi_{1}(t, q)-L_{0}(t)\right] & =q h\left\{\mathcal{N}_{1}\left(\phi_{1}(t, q)\right)-g_{1}(t)\right\} \\
(1-q) \mathcal{L}\left[\phi_{2}(t, q)-D_{0}(t)\right] & =q h\left\{\mathcal{N}_{2}\left(\phi_{2}(t, q)\right)-g_{2}(t)\right\}
\end{aligned}
$$

where $q \in[0,1]$ is an embedding parameter, $h$ is a nonzero auxiliary function, $\mathcal{L}$ is an auxiliary linear operator, $N_{1,0}(t), N_{2,0}(t)$ are the initial guesses of $N_{1}(t), N_{2}(t)$, and $\phi_{i}(t, q)$ are unknown functions.

\subsubsection{Solution as polynomial functions}

Now, by taking polynomials as base functions the $m^{\text {th }}$ order deformation equations are given by

$$
\begin{aligned}
& \mathcal{L}\left[N_{1, m}(t)-N_{1, m-1}(t)\right]=q h R_{1, m}\left(\vec{N}_{1, m-1}\right) \\
& \mathcal{L}\left[N_{2, m}(t)-N_{2, m-1}(t)\right]=q h R_{2, m}\left(\vec{N}_{2, m-1}\right)
\end{aligned}
$$


As $q$ increases from 0 to 1 , the solutions $\phi_{1}(t, q), \phi_{2}(t, q)$ vary from the initial guesses $N_{1,0}(t), N_{2,0}(t)$ to the solutions $N_{1}(t), N_{2}(t)$. where

$$
\begin{aligned}
& R_{1, m}\left(\vec{N}_{1, m}\right)=\dot{N}_{1, m-1}-a_{1} N_{1, m-1}(t)+ \\
& b_{11} \sum_{i=0}^{m-1} N_{1, i}(t) N_{1, m-1-i}(t)+b_{12} \sum_{i=0}^{m-1} N_{1, i}(t) N_{2, m-1-i}(t) \\
& R_{2, m}\left(\vec{N}_{2, m}\right)=\dot{N}_{2, m-1}-a_{2} N_{2, m-1}(t)+ \\
& b_{21} \sum_{i=0}^{m-1} N_{2, i}(t) N_{1, m-1-i}(t)+b_{22} \sum_{i=0}^{m-1} N_{2, i}(t) N_{2, m-1-i}(t)
\end{aligned}
$$

subject to $N_{1, m}=0, N_{2, m}=0$

where the dot denotes differentiation with respect to the variable $t$. The solution of the $m^{\text {th }}$ order deformation equation for $m \geq 1$ is given by

$$
\begin{aligned}
& N_{1, m}(t)=\chi_{2} N_{1, m-1}(t)+h \mathcal{L}^{-1}\left[R_{1, m}\left(\vec{N}_{1, m-1}\right)\right] \\
& N_{2, m}(t)=\chi_{2} N_{2, m-1}(t)+h \mathcal{L}^{-1}\left[R_{2, m}\left(\vec{N}_{2, m-1}\right)\right] \\
& \text { and } \chi_{m}=\left\{\begin{array}{c}
0, m \leq 1 \\
1, \text { otherwise }
\end{array}\right.
\end{aligned}
$$

The integration constants $c_{i}$ are determined by the initial condition. Now the successive approximations are given by

$$
\begin{aligned}
& N_{1,1}(t)=\left[-a_{1}+b_{11}+2 b_{12}\right] h t \\
& N_{2,1}(t)=\left[-2 a_{2}+2 b_{21}+4 b_{22}\right] h t \\
& N_{1,2}(t)=\left[-a_{1}+b_{11}+2 b_{12}\right] h t+\left[-a_{1}+b_{11}+2 b_{12}\right] h^{2} t+\left\{\left[-a_{1}\left[-a_{1}+b_{11}+2 b_{12}\right]+\right.\right. \\
& \left.2\left[-a_{1}+b_{11}+2 b_{12}\right] b_{11}+b_{12}\left[2\left[-a_{2}+b_{21}+2 b_{22}\right]+2\left[-a_{1}+b_{11}+2 b_{12}\right]\right]\right\}\left[\left(h^{2} t^{2} / 2\right)\right. \\
& N_{2,2}(t)=\left[-2 a_{2}+2 b_{21}+4 b_{22}\right] h t+\left[-2 a_{2}+2 b_{21}+4 b_{22}\right] h^{2} t+\left\{\left[-a_{2}\left[-2 a_{2}+2 b_{21}+4 b_{22}\right]+\right.\right. \\
& \left.\left[-8 a_{2}+8 b_{21}+16 b_{22}\right] b_{12}+b_{21}\left[2\left[-a_{1}+b_{11}+2 b_{12}\right]+\left[-2 a_{2}+2 b_{21}+4 b_{22}\right]\right]\right\}\left[\left(h^{2} t^{2} / 2\right)\right.
\end{aligned}
$$

Now the analytic solution via the polynomial base functions of the system has the general form

$$
N_{1}(t)=\sum_{m=1}^{\infty} a_{i, m}(h) t^{m}, \quad N_{2}(t)=\sum_{m=1}^{\infty} a_{i, m}^{\prime}(h) t^{m}
$$

The above expressions represent a family of solution expressions in the auxiliary parameter $h$. By using $h$-curves [16] valid regions of a convergent solution series can be determined. 


\subsubsection{Solution as exponential functions}

Now, by taking exponential functions as base functions, the initial approximations of (33),(34) satisfying the initial conditions

$$
N_{1}(0)=1, N_{2}(0)=2
$$

and the auxiliary linear operators $\mathcal{L}$ for $i=1,2,3$ are

$$
\mathcal{L}\left[\phi_{i}(t, q)\right]=\frac{\left.\partial \phi_{i}(t, q)\right)}{\partial t}+\phi_{i}(t, q), i=1,2, \text { with } \mathcal{L}\left(\left[C_{i} e^{-t}\right]=0 .\right.
$$

The solution of the $m^{\text {th }}$ order deformation equation for $m \geq 1$ is given by

$$
\begin{aligned}
& N_{1, m}(t)=\chi_{2} N_{1, m-1}(t)+h e^{-t} \int_{0}^{t} e^{\tau} H(\tau) R_{m}\left(\vec{N}_{1, m-1}\right) d \tau+C_{1} e^{-t} \\
& N_{2, m}(t)=\chi_{2} N_{2, m-1}(t)+h e^{-t} \int_{0}^{t} e^{\tau} H(\tau) R_{m}\left(\vec{N}_{2, m-1}\right) d \tau+C_{2} e^{-t} \\
& \text { and } \chi_{m}=\left\{\begin{array}{c}
0, m \leq 1 \\
1, \text { otherwise }
\end{array}\right.
\end{aligned}
$$

The integration constants $c_{i}$ are determined by the initial condition. Now the successive approximations obtained by taking $H(\tau)=1$ in the above equations are given by

$$
\begin{aligned}
& N_{1,1}(t)=\left[-a_{1}+b_{11}+2 b_{12}\right] h\left(1-e^{-t}\right) \\
& N_{2,1}(t)=\left[-2 a_{2}+2 b_{21}+4 b_{22}\right] h\left(1-e^{-t}\right) \\
& N_{1,2}(t)=\left[-a_{1}+b_{11}+2 b_{12}\right] h\left(1-e^{-t}\right)+\left[-a_{1}+b_{11}+2 b_{12}\right] h^{2} t e^{-t} \\
& \left\{-a_{1}\left[-a_{1}+b_{11}+2 b_{12}\right]+2 b_{11}\left[-a_{1}+b_{11}+2 b_{12}+b_{12}\left[\left[-2 a_{2}+2 b_{21}\right.\right.\right.\right. \\
& \left.\left.\left.\left.+4 b_{22}\right]+2\left[-a_{1}+b_{11}+2 b_{12}\right]\right]\right]\right\}\left[h^{2}\left(e^{-t}+t-1\right)\right] \\
& N_{2,2}(t)=\left[-2 a_{2}+2 b_{21}+4 b_{22}\right] h\left(1-e^{-t}\right)+\left[-2 a_{2}+2 b_{21}+4 b_{22}\right] h^{2} t e^{-t} \\
& \left\{-a_{2}\left[-2 a_{2}+2 b_{21}+4 b_{22}\right]+4 b_{12}\left[-2 a_{2}+2 b_{21}+4 b_{22}\right]+b_{21}\left[\left[-2 a_{2}+2 b_{21}\right.\right.\right. \\
& \left.\left.\left.\left.+4 b_{22}\right]+2\left[-a_{1}+b_{11}+2 b_{12}\right]\right]\right]\right\}\left[h^{2}\left(e^{-t}+t-1\right)\right]
\end{aligned}
$$

Now the analytic solution via the polynomial base functions of the system has the general form

$$
N_{1}(t)=\sum_{m=1}^{\infty} N_{1, m}(t), N_{2}(t)=\sum_{m=1}^{\infty} N_{2, m}(t)
$$

By using $h$-curves [16] valid regions of a convergent solution series can be determined.

\section{Three species ecological system}

We consider a three species ecological system

$$
\begin{aligned}
\dot{L}(t) & =L(t)\left[P_{1}-\gamma D(t)+\beta W(t)\right] \\
\dot{D}(t) & =D(t)\left[P_{2}+\gamma L(t)-\alpha W(t)\right] \\
\dot{W}(t) & =W(t)\left[P_{3}-\beta L(t)+\alpha D(t)\right]
\end{aligned}
$$

where $L(t), D(t)$ and $W(t)$ denote the amount of litter, detritus and predators in the sea at time $t$ in the mangrove area respectively, $\alpha, \beta, \gamma, P_{1}, P_{2}, P_{3}$ denote the parameters. 


\subsection{Three species system - perturbation method}

We making the following assumptions (i) $\mathrm{W}(\mathrm{t})$ preys on $\mathrm{D}(\mathrm{t}), \mathrm{D}(\mathrm{t})$ preys on $\mathrm{L}(\mathrm{t})$ and $\mathrm{L}(\mathrm{t})$ preys on $\mathrm{W}(\mathrm{t})(\mathrm{ii}) \mathrm{W}(\mathrm{t})$ preys on $\mathrm{D}(\mathrm{t})$ and $\mathrm{L}(\mathrm{t})$, in the absence of litter and detritus population of coastal organisms decrease. $\mathrm{D}(\mathrm{t})$ preys on $\mathrm{L}(\mathrm{t}), \mathrm{L}(\mathrm{t})$ has sufficient bio chemicals, so in the absence of detritus and predators Litter increases without limit.ie., (i) $\alpha, \beta, \gamma>0$ (ii) $\alpha, \gamma>0, \beta<0, P_{1}>$ $0, P_{3}>0$ and

$$
P_{1} \alpha+P_{2} \beta+P_{3} \gamma=0
$$

The equilibrium point for the system (94) is calculated by using the concept of generalized inverse [2] and is given by solving the following system

$$
P_{1}=\gamma D^{*}(t)-\beta W^{*}(t), P_{2}=-\gamma L^{*}(t)+\alpha W^{*}(t), P_{3}=\beta L^{*}(t)-\alpha D^{*}(t)
$$

By solving the above system using algorithm [2] for the generalized inverse the equilibrium point of (94) is given by

$$
L^{*}=\frac{P_{3} \beta-P_{2} \gamma}{\alpha^{2}+\beta^{2}+\gamma^{2}}, D^{*}=\frac{P_{1} \gamma-P_{3} \alpha}{\alpha^{2}+\beta^{2}+\gamma^{2}}, W^{*}=\frac{P_{2} \alpha-P_{1} \beta}{\alpha^{2}+\beta^{2}+\gamma^{2}}
$$

Now we define the new densities deviating from the equilibrium point as

$$
L_{1}(t)=L(t)-L^{*}, D_{1}(t)=D(t)-D^{*}, W_{1}(t)=W(t)-W^{*}
$$

Now the system of equations (94)become

$$
\begin{gathered}
\dot{L}_{1}(t)=-\gamma L_{1}^{*} D_{1}(t)+\beta L_{1}^{*} W_{1}(t)-\gamma L_{1}(t) D_{1}(t)+\beta L_{1}(t) W_{1}(t) \\
\dot{D}_{1}(t)=\gamma D^{*} L_{1}(t)-\alpha D^{*} W_{1}(t)+\gamma L_{1}(t) D_{1}(t)-\alpha D_{1}(t) W_{1}(t) \\
\dot{W}(t)_{1}=-\beta W^{*} L_{1}(t)+\alpha W^{*} D_{1}(t)-\beta L_{1}(t) W_{1}(t)+\alpha D_{1}(t) W_{1}(t)
\end{gathered}
$$

We introduce the parameter $\mu$ in to the non linear terms of the above system we get the following

$$
\begin{gathered}
\dot{L}_{1}(t)=-\gamma L^{*} D_{1}(t)+\beta L^{*} W_{1}(t)-\mu \gamma L_{1}(t) D_{1}(t)+\mu \beta L_{1}(t) W_{1}(t) \\
\dot{D}_{1}(t)=\gamma D^{*} L_{1}(t)-\alpha D^{*} W_{1}(t)+\mu \gamma L_{1}(t) D_{1}(t)-\mu \alpha D_{1}(t) W_{1}(t) \\
\dot{W}_{1}(t)=-\beta W^{*} L_{1}(t)+\alpha W^{*} D_{1}(t)-\mu \beta L_{1}(t) W_{1}(t)+\mu \alpha D_{1}(t) W_{1}(t)
\end{gathered}
$$

We seek solutions of the (100) in the form

$$
\begin{gathered}
L_{1}(t)=L_{1_{0}}(t)+\mu L_{1_{1}}(t)+\mu^{2} L_{1_{2}}(t)+\ldots \\
D_{1}(t)=D_{1_{0}}(t)+\mu D_{1_{1}}(t)+\mu^{2} D_{1_{2}}(t)+\ldots \\
W_{1}(t)=W_{1_{0}}(t)+\mu W_{1_{1}}(t)+\mu^{2} W_{1_{2}}(t)+\ldots
\end{gathered}
$$

By equating like powers of $\mu$ after substituting (101) in (100) we get

$$
\begin{aligned}
& L_{1_{0}}(t)=-\gamma L^{*} D_{1_{0}}(t)+\beta L^{*} W_{1_{0}}(t) \\
& D_{1_{0}}(t)=\gamma L D^{*} L_{1_{0}}(t)-\alpha D^{*} W_{1_{0}}(t) \\
& W_{1_{0}}(t)=-\beta W^{*} L_{1_{0}}(t)+\alpha W^{*} D_{1_{0}}(t)
\end{aligned}
$$




$$
\begin{aligned}
& L_{1_{1}}(t)=-\gamma L^{*} D_{1_{1}}(t)+\beta L^{*} W_{1_{1}}(t)-\gamma L_{1_{0}} D_{1_{0}}(t)+\beta L_{1_{0}} W_{1_{0}}(t) \\
& D_{1_{1}}(t)=\gamma L D^{*} L_{1_{1}}(t)-\alpha D^{*} W_{1_{1}}(t)+\gamma L_{1_{0}} D_{1_{0}}(t)-\alpha D_{1_{0}} W_{1_{0}}(t) \\
& W_{1_{1}}(t)=-\beta W^{*} L_{1_{1}}(t)+\alpha W^{*} D_{1_{1}}(t)-\beta L_{1_{0}} W_{1_{0}}(t)+\alpha D_{1_{0}} W_{1_{0}}(t)
\end{aligned}
$$

The solution of the equation (102) with the initial condition $L_{1}(0)=C_{1}, D_{1}(0)=C_{2}, W_{1}(0)=$ $C_{3}$ by using Laplace transformation and the generating functions $L_{1}(t), D_{1}(t), W_{1}(t)$ is given by

$$
\begin{aligned}
& L_{1_{0}}(t)=\left(C_{1}-\frac{Q_{1}}{K_{1}^{2}}\right) \operatorname{Cos}\left(K_{1} t\right)+\left(\frac{C_{3} L^{*} \beta-C_{2} L^{*} \gamma}{k_{1}}\right) \operatorname{Sin}\left(k_{1} t\right)+\frac{Q_{1}}{k_{1}^{2}} \\
& D_{1_{0}}(t)=\left(C_{2}-\frac{Q_{2}}{K_{1}^{2}}\right) \operatorname{Cos}\left(K_{1} t\right)+\left(\frac{C_{1} D^{*} \gamma-C_{3} D^{*} \alpha}{k_{1}}\right) \operatorname{Sin}\left(k_{1} t\right)+\frac{Q_{2}}{k_{1}^{2}} \\
& W_{1_{0}}(t)=\left(C_{3}-\frac{Q_{3}}{K_{1}^{2}}\right) \operatorname{Cos}\left(K_{1} t\right)+\left(\frac{C_{2} W^{*} \alpha-C_{1} W^{*} \beta}{k_{1}}\right) \operatorname{Sin}\left(k_{1} t\right)+\frac{Q_{3}}{k_{1}^{2}}
\end{aligned}
$$

where

$k_{1}^{2}=L^{*} D^{*} \gamma^{2}+L^{*} W^{*} \beta^{2}+D^{*} W^{*} \alpha^{2}, Q_{1}=C_{1} D^{*} W^{*} \alpha^{2}+C_{2} L^{*} W^{*} \alpha \beta$

$+C_{3} L^{*} D^{*} \alpha \gamma, Q_{2}=C_{1} D^{*} W^{*} \alpha \beta+C_{2} L^{*} W^{*} \beta^{2}+C_{3} L^{*} D^{*} \beta \gamma, Q_{3}=$

$C_{1} D^{*} W^{*} \alpha \gamma+C_{2} L^{*} W^{*} \beta \gamma+C_{3} L^{*} D^{*} \gamma^{2} ; C_{1}, C_{2}$ and $C_{3}$ are the initial values of $L_{1_{0}}(t), D_{1_{0}}(t)$ and $W_{1_{0}}(t)$ respectively. Now the solutions of (103) with the initial condition $L_{1}(0)=$ $0, D_{1}(0)=0, W_{1}(0)=0$ are given by

$$
\begin{aligned}
L_{1_{1}}(t)= & \frac{A_{1}}{2 K_{1}} \operatorname{Sin}\left(2 K_{1} t\right)+A_{2} \operatorname{Cos}\left(2 k_{1} t\right)+\left\{\frac{A_{3}}{k_{1}}+\frac{A_{5}}{2 k_{1}^{3}}+\frac{A_{6} t}{2 k_{1}}\right\} \operatorname{Sin}\left(k_{1} t\right)+\left\{A_{4}+\frac{A_{5} t}{2 k_{1}^{2}}\right\} \\
& \operatorname{Cos}\left(k_{1} t\right)+A_{7} \\
D_{1_{1}}(t)= & \frac{B_{1}}{2 K_{1}} \operatorname{Sin}\left(2 K_{1} t\right)+B_{2} \operatorname{Cos}\left(2 k_{1} t\right)+\left\{\frac{B_{3}}{k_{1}}+\frac{B_{5}}{2 k_{1}^{3}}+\frac{B_{6} t}{2 k_{1}}\right\} \operatorname{Sin}\left(k_{1} t\right)+\left\{B_{4}+\frac{B_{5} t}{2 k_{1}^{2}}\right\} \\
& \operatorname{Cos}\left(k_{1} t\right)+B_{7} \\
W_{1_{1}}(t)= & \frac{D_{1}}{2 K_{1}} \operatorname{Sin}\left(2 K_{1} t\right)+D_{2} \operatorname{Cos}\left(2 k_{1} t\right)+\left\{\frac{D_{3}}{k_{1}}+\frac{D_{5}}{2 k_{1}^{3}}+\frac{D_{6} t}{2 k_{1}}\right\} \operatorname{Sin}\left(k_{1} t\right)+\left\{D_{4}+\frac{D_{5} t}{2 k_{1}^{2}}\right\} \\
& \operatorname{Cos}\left(k_{1} t\right)+D_{7}
\end{aligned}
$$

where $A_{1}=\frac{4 R_{1}}{3}-\frac{X_{2}}{3 k_{1}^{2}}+\frac{X_{3}}{12 k_{1}^{4}}, A_{2}=-\frac{X_{1}}{3 k_{1}^{2}}, A_{3}=R_{3}-\frac{R_{1}}{3}+\frac{X_{2}}{3 k_{1}^{2}}-\frac{X_{3}}{3 k_{1}^{4}}, A_{4}=\frac{X_{1}}{3 k_{1}^{2}}-\frac{X_{6}}{k_{1}^{4}}, A_{5}=$ $X_{5}-R_{3} k_{1}^{2}, A_{6}=X_{4}-\frac{X_{6}}{k_{1}^{2}}, A_{7}=\frac{X_{3}}{4 k_{1}^{4}}+\frac{X_{6}}{k_{1}^{4}}$,

$B_{1}=\frac{4 S_{1}}{3}-\frac{Y_{2}}{3 k_{1}^{2}}+\frac{Y_{3}}{12 k_{1}^{4}}, B_{2}=-\frac{Y_{1}}{3 k_{1}^{2}}, B_{3}=S_{3}-\frac{S_{1}}{3}+\frac{Y_{2}}{3 k_{1}^{2}}-\frac{Y_{3}}{3 k_{1}^{4}}, B_{4}=\frac{Y_{1}}{3 k_{1}^{2}}-\frac{Y_{6}}{k_{1}^{4}}, B_{5}=$ $Y_{5}-S_{3} k_{1}^{2} B_{6}=Y_{4}-\frac{Y_{6}}{k_{1}^{2}}, B_{7}=\frac{Y_{3}}{4 k_{1}^{4}}+\frac{Y_{6}}{k_{1}^{4}}$,

$D_{1}=\frac{4 T_{1}}{3}-\frac{Z_{2}}{3 k_{1}^{2}}+\frac{Z_{3}}{12 k_{1}^{4}}, D_{2}=-\frac{Z_{1}}{3 k_{1}^{2}}, D_{3}=T_{3}-\frac{T_{1}}{3}+\frac{Z_{2}}{3 k_{1}^{2}}-\frac{Z_{3}}{3 k_{1}^{4}}, D_{4}=\frac{Z_{1}}{3 k_{1}^{2}}-\frac{Z_{6}}{k_{1}^{4}}, D_{5}=$ $Z_{5}-T_{3} k_{1}^{2}, D_{6}=Z_{4}-\frac{Z_{6}}{k_{1}^{2}}, D_{7}=\frac{Z_{3}}{4 k_{1}^{4}}+\frac{Z_{6}}{k_{1}^{4}}$

where $X_{1}=2 K_{1} R_{2}-S_{1} \gamma L^{*}-T_{1} L^{*} \beta, X_{2}=\alpha^{2} D^{*} W^{*} R_{1}-2 k_{1} S_{2} \gamma L^{*}+T_{1} \alpha \gamma L^{*} D^{*}+$ 
$2 k_{1} T_{2} \beta L^{*}, X_{3}=2 k_{1} R_{2} \alpha^{2} D^{*} W^{*}+2 k_{1} T_{2} \alpha \gamma L^{*} D^{*}+2 k_{1} S_{2} \alpha \beta L^{*} W^{*}, X_{4}=k_{1} R_{4}-S_{3} \gamma L^{*}+$ $T_{3} \beta L^{*}, X_{5}=R_{3} \alpha^{2} D^{*} W^{*}-k_{1} S_{4} \gamma L^{*}+T_{3} \alpha \gamma L^{*} W^{*}+S_{3} \alpha \beta L^{*} W^{*}+k_{1} T_{4} \beta L^{*}, X_{6}=$ $k_{1} R_{4} \alpha^{2} D^{*} W^{*}+k_{1} T_{4} \alpha \gamma L^{*} D^{*}+k_{1} S_{4} \alpha \beta L^{*} W^{*}$,

$Y_{1}=2 K_{1} S_{2}-T_{1} \alpha D^{*}+R_{1} D^{*} \gamma, Y_{2}=2 k_{1} R_{2} \gamma D^{*}-2 k_{1} T_{2} \alpha D^{*}+R_{1} \alpha \beta D^{*} W^{*}+T_{1} \beta \gamma L^{*} D^{*}+$ $S_{1} \beta^{2} L^{*} W^{*}, Y_{3}=2 k_{1} R_{2} \alpha \beta D^{*} W^{*}+2 k_{1} T_{2} \beta \gamma L^{*} D^{*}+2 k_{1} S_{2} \beta^{2} L^{*} W^{*}, Y_{4}=k_{1} S_{4}-T_{3} \alpha D^{*}+$ $R_{3} \gamma D^{*}, \quad Y_{5}=R_{4} k_{1} \gamma D^{*}+R_{3} \alpha \beta D^{*} W^{*}-T_{4} k_{1} \alpha D^{*}+T_{3} \gamma \beta L^{*} D^{*}+S_{3} \beta^{2} L^{*} W^{*}, Y_{6}=$ $k_{1} R_{4} \alpha \beta D^{*} W^{*}+k_{1} T_{4} \beta \gamma L^{*} D^{*}+k_{1} S_{4} \beta^{2} L^{*} W^{*}$,

$Z_{1}=2 K_{1} T_{2}+S_{1} \alpha W^{*}-R_{1} W^{*} \beta, Z_{2}=2 k_{1} S_{2} \alpha W^{*}+T_{1} \gamma^{2} L^{*} D^{*}+S_{1} \gamma \beta L^{*} W^{*}+R_{1} \alpha \gamma W^{*} D^{*}-$ $2 k_{1} R_{2} \beta W^{*}, Z_{3}=2 k_{1} T_{2} \gamma^{2} D^{*} L^{*}+2 k_{1} S_{2} \beta \gamma L^{*} W^{*}+2 k_{1} R_{2} \alpha \gamma D^{*} W^{*}, Z_{4}=k_{1} T_{4}+S_{3} \alpha W^{*}-$ $R_{3} \beta W^{*}, Z_{5}=S_{4} k_{1} \alpha W^{*}+T_{3} \gamma^{2} D^{*} L^{*}+S_{3} k_{1} \gamma \beta L^{*} W^{*}+R_{3} \alpha \gamma W^{*} D^{*}-k_{1} R_{4} \beta W^{*}, Z_{6}=$ $k_{1} T_{4} \gamma^{2} D^{*} L^{*}+k_{1} S_{4} \beta \gamma L^{*} W^{*}+k_{1} R_{4} \alpha \gamma D^{*} W^{*}$,

$R_{1}=\left(C_{1}-\frac{Q_{1}}{k_{1}^{2}}\right)\left(C_{3} \beta-C_{2} \gamma\right), R_{2}=\frac{L^{* 2} k_{1}^{2}\left(C_{3} \beta-C_{2} \gamma\right)^{2}-\left(C_{1} k_{1}^{2}-Q_{1}\right)^{2}}{2 L^{*} k_{1}^{3}}, R_{3}=\frac{Q_{1}}{k_{1}^{2}}\left(C_{3} \beta-C_{2} \gamma\right), R_{4}=$ $\frac{Q_{1}}{L^{*} k_{1}}\left(\frac{Q_{1}}{k_{1}^{2}}-C_{1}\right), S_{1}=\left(C_{2}-\frac{Q_{2}}{k_{1}^{2}}\right)\left(C_{1} \gamma-C_{3} \alpha\right), S_{2}=\frac{D^{* 2} k_{1}^{2}\left(C_{1} \gamma-C_{3} \alpha\right)^{2}-\left(C_{2} k_{1}^{2}-Q_{2}\right)^{2}}{2 D^{*} k_{1}^{3}}, S_{3}=\frac{Q_{2}}{k_{1}^{2}}\left(C_{1} \gamma-\right.$ $\left.C_{3} \alpha\right), S_{4}=\frac{Q_{2}}{D^{*} k_{1}}\left(\frac{Q_{2}}{k_{1}^{2}}-C_{2}\right), T_{1}=\left(C_{3}-\frac{Q_{3}}{k_{1}^{2}}\right)\left(C_{2} \alpha-C_{1} \beta\right), T_{2}=\frac{W^{* 2} k_{1}^{2}\left(C_{2} \alpha-C_{1} \beta\right)^{2}-\left(C_{3} k_{1}^{2}-Q_{3}\right)^{2}}{2 W^{*} k_{1}^{3}}, T_{3}=$ $\frac{Q_{3}}{k_{1}^{2}}\left(C_{2} \alpha-C_{1} \beta\right), T_{4}=\frac{Q_{3}}{W^{*} k_{1}}\left(\frac{Q_{3}}{k_{1}^{2}}-C_{3}\right)$.

Now the solution of (99) is given by $L_{1}(t) \simeq L_{1_{0}}(t)+L_{1_{1}}(t), D_{1}(t) \simeq D_{1_{0}}(t)+D_{1_{1}}(t), W_{1}(t) \simeq W_{1_{0}}(t)+W_{1_{1}}(t)$

Now the solution of the three species ecological system (94) is given by

$L=\frac{P_{3} \beta-P_{2} \gamma}{\alpha^{2}+\beta^{2}+\gamma^{2}}+\left(C_{1}-\frac{Q_{1}}{K_{1}^{2}}+A_{4}+\frac{A_{5} t}{2 k_{1}^{2}}\right) \operatorname{Cos}\left(K_{1} t\right)+\left(\frac{C_{3} L^{*} \beta-C_{2} L^{*} \gamma}{k_{1}}+\frac{A_{3}}{k_{1}}+\frac{A_{5}}{2 k_{1}^{3}}+\frac{A_{6} t}{2 k_{1}}\right) \operatorname{Sin}\left(k_{1} t\right)+$ $\frac{A_{1}}{2 K_{1}} \operatorname{Sin}\left(2 K_{1} t\right)+A_{2} \operatorname{Cos}\left(2 k_{1} t\right)+\frac{Q_{1}}{k_{1}^{2}}+A_{7}$

$D=\frac{P_{1} \gamma-P_{3} \alpha}{\alpha^{2}+\beta^{2}+\gamma^{2}}+\left(C_{2}-\frac{Q_{2}}{K_{1}^{2}}+B_{4}+\frac{B_{5} t}{2 k_{1}^{2}}\right) \operatorname{Cos}\left(K_{1} t\right)+\left(\frac{C_{1} D^{*} \gamma-C_{3} D^{*} \alpha}{k_{1}}+\frac{B_{3}}{k_{1}}+\frac{B_{5}}{2 k_{1}^{3}}+\frac{B_{6} t}{2 k_{1}}\right) \operatorname{Sin}\left(k_{1} t\right)+$ $\frac{B_{1}}{2 K_{1}} \operatorname{Sin}\left(2 K_{1} t\right)+B_{2} \operatorname{Cos}\left(2 k_{1} t\right)+\frac{Q_{2}}{k_{1}^{2}}+B_{7}$

$\left.W=\frac{P_{2} \alpha-P_{1} \beta}{\alpha^{2}+\beta^{2}+\gamma^{2}}+C_{3}-\frac{Q_{3}}{K_{1}^{2}}+D_{4}+\frac{D_{5} t}{2 k_{1}^{2}}\right) \operatorname{Cos}\left(K_{1} t\right)+\left(\frac{C_{2} W^{*} \alpha-C_{1} W^{*} \beta}{k_{1}}+\frac{D_{3}}{k_{1}}+\frac{D_{5}}{2 k_{1}^{3}}+\frac{D_{6} t}{2 k_{1}}\right) \operatorname{Sin}\left(k_{1} t\right)+$ $\frac{D_{1}}{2 K_{1}} \operatorname{Sin}\left(2 K_{1} t\right)+D_{2} \operatorname{Cos}\left(2 k_{1} t\right)+\frac{Q_{3}}{k_{1}^{2}}+D_{7}$.

These solutions are valid for larger deviations from the equilibrium point since the additional correction terms improves accuracy. By taking the transformation

$$
L=L_{1}+L^{*}, D=D_{1}+D^{*}, W=W_{1}+W^{*}
$$

where $L^{*}, D^{*}$ and $W^{*}$ are the equilibrium points given by (97), the three species ecological system becomes

$$
\begin{aligned}
\dot{L}_{1} & =\left[L^{*}+L_{1}\right]\left[-\gamma D_{1}+\beta W_{1}\right] \\
\dot{D}_{1} & =\left[D^{*}+D_{1}\right]\left[-\alpha W_{1}+\gamma L_{1}\right] \\
\dot{W}_{1} & =\left[W^{*}+W_{1}\right]\left[-\beta L_{1}+\alpha D_{1}\right]
\end{aligned}
$$

By defining a Liapunov function $V(t)$, positive definite function as

$$
\begin{array}{r}
V(t)=d_{1}\left[L_{1}-L^{*} \log \left(1+L_{1} / L^{*}\right)\right]+d_{2}\left[D_{1}-D^{*} \log \left(1+D_{1} / D^{*}\right)\right]+ \\
d_{3}\left[W_{1}-W^{*} \log \left(1+W_{1} / W^{*}\right)\right]
\end{array}
$$


with $d_{1}, d_{2}, d_{3}$ as positive constants, $\left[d_{2}-d_{1}\right] \gamma \leq 0,\left[d_{3}-d_{2}\right] \alpha \leq 0$ and $\left[d_{1}-d_{3}\right] \beta \leq 0$ we can observe that the three species ecological system is asymptotically stable since

$$
\dot{V}(t)=d_{1} L_{1}\left[-\gamma D_{1}+\beta W_{1}\right]+d_{2} D_{1}\left[-\alpha W_{1}+\gamma L_{1}\right]+d_{3} W_{1}\left[-\beta L_{1}+\alpha D_{1}\right] \leq 0
$$

\subsection{Three species system - Adomian decomposition method}

It is a well recognized fact that the enormous production of mangrove detritus provides a necessary energy to drive the biological machinery of mangrove estuarine ecosystem besides supporting to certain extent the coastal population. In the process of litter decomposition, if the food material is not ingested by other animals, the decomposition process of litter completes and this liberates nutrients into water and soil. Part of the detritus produced in mangrove estuarine ecosystem may be carried to the adjacent coastal waters through tidal fluxes. In fact here detritus serves a couple of purposes. (i) Regenerates nutrients by undergoing complete decomposition process and (ii) Serves as a food source to estuarine and coastal organisms like Polychaete, Crabs, Prawn, Fish and Zooplankton. Here after we call all these consumers as predators. Now we are in a position to formulate the mathematical model. If $L(t), D(t)$ and $W(t)$ denote the amount of litter, detritus and predators in the sea at time $t$ in the mangrove area respectively then the following assumptions are reasonable. (1) If there is no litter from the mangroves the detritus formation decreases in a way proportional to litter and if there is no detritus formation, the amount of litter increases exponentially within a limited time. (2) If there are no bacteria, fungi and protozoa, the formation of detritus decreases and the amount of litter increases in a limited time and (3) In The absence of consumers like lower Carnivores and higher Carnivores, fish etc... the amount of detritus increases and in the absence of detritus in the sea, the growth rate of above predators decreases. These assumptions give rise to the following system of non linear first order differential equations,

$$
\dot{L}=\alpha_{1} L-\beta_{1} L D, \dot{D}=\alpha_{2} D+\beta_{2} L D-\gamma_{2} D W, \dot{W}=-\alpha_{3} W+\gamma_{3} D W
$$

where $\alpha_{i}(i=1,2,3), \beta_{j}(j=1,2)$ and $\gamma_{k}(k=2,3)$ are all positive constants. The presence of both litter and detritus is beneficial to the growth of predators like fish and carnivores in the sea. More specifically the predator species increases and the detritus decreases at rates proportional to the product of the two. If they also satisfy the condition,

$$
\alpha_{1} \gamma_{3}-\alpha_{3} \beta_{1}=0
$$

It amounts to saying that the growth of predators is directly proportional to the formation of the detritus. The interior equilibrium point for the system (108) is calculated by pseudo inverse concept [2] and on solving the system of equations

$$
\beta_{1} D=\alpha_{1}, \quad-\beta_{2} L+\gamma_{2} W=\alpha_{2}, \quad \gamma_{3} D=\alpha_{3}
$$

The possible equilibria are (i) Trivial equilibrium point $L=0, D=0$ and $W=0$. (ii) Equilibrium in the absence of predators $L_{w}=-\alpha_{2} / \beta_{2}, D_{W}=\alpha_{1} / \beta_{1}$ (iii) Equilibrium in the absence of Detritus $L_{D}=0, W_{D}=0$ (iv) Equilibrium in the absence of Litter $D_{L}=\alpha_{3} / \gamma_{3}$ , $W_{L}=\alpha_{2} / \gamma_{2}$ and (v)The interior equilibrium

$$
L=-\left(\alpha_{2} \beta_{2}\right) /\left(\beta_{2}^{2}+\gamma_{2}^{2}\right), D=\left(\alpha_{1} \beta_{1}+\alpha_{3} \gamma_{3}\right) /\left(\beta_{1}^{2}+\gamma_{3}^{2}\right), W=\left(\alpha_{2} \gamma_{2}\right) /\left(\beta_{2}^{2}+\gamma_{2}^{2}\right)
$$


Equilibrium (ii) is the case when the entire litter is decomposed in to detritus which is usual prey predator equilibrium, whereas (iii) is the equilibrium with no detritus and equilibrium (iv) is the one with no predators. Neither all these three equilibria (ii), (iii) and (iv) nor the trivial equilibrium are of much interest to us. Define densities of the three species deviating from the interior equilibrium values given in (111) as

$$
L_{1}=L-a, D_{1}=D-b, W_{1}=W-c
$$

where $a=-\left(\alpha_{2} \beta_{2}\right) /\left(\beta_{2}^{2}+\gamma_{2}^{2}\right), b=\left(\alpha_{1} \beta_{1}+\alpha_{3} \gamma_{3}\right) /\left(\beta_{1}^{2}+\gamma_{3}^{2}\right)$ and $c=\left(\alpha_{2} \gamma_{2}\right) /\left(\beta_{2}^{2}+\gamma_{2}^{2}\right)$. From equation (108) we get

$$
\begin{aligned}
\dot{L_{1}} & =-a \beta_{1} D_{1}-\beta_{1} L_{1} D_{1} \\
\dot{D}_{1} & =b \beta_{2} L_{1}-b \gamma_{2} W_{1}+\beta_{2} L_{1} D_{1}-\gamma_{2} D_{1} W_{1} \\
\dot{W}_{1} & =c \gamma_{3} D_{1}+\gamma_{3} D_{1} W_{1}
\end{aligned}
$$

The above equations can be represented by the system

$$
\dot{X}=E_{1} X B+E_{2} X C+E_{1} X F_{1} X+E_{2} X F_{2} X
$$

Where $X=\left[\begin{array}{ccc}L_{1} & 0 & 0 \\ 0 & D_{1} & 0 \\ 0 & 0 & W_{1}\end{array}\right], E_{1}=\left[\begin{array}{lll}0 & 1 & 0 \\ 0 & 0 & 1 \\ 1 & 0 & 0\end{array}\right], E_{2}=\left[\begin{array}{lll}0 & 0 & 1 \\ 1 & 0 & 0 \\ 0 & 1 & 0\end{array}\right], B=\left[\begin{array}{ccc}0 & 0 & 0 \\ -a \beta_{1} & 0 & 0 \\ 0 & -b \gamma_{2} & 0\end{array}\right]$,

$C=\left[\begin{array}{ccc}0 & b \beta_{2} & 0 \\ 0 & 0 & c \gamma_{3} \\ 0 & 0 & 0\end{array}\right], F_{1}=\left[\begin{array}{ccc}0 & 0 & 0 \\ -\beta_{1} & 0 & 0 \\ 0 & -\gamma_{2} & 0\end{array}\right], F_{2}=\left[\begin{array}{ccc}0 & \beta_{2} & 0 \\ 0 & 0 & \gamma_{3} \\ 0 & 0 & 0\end{array}\right]$. The initial conditions $L(0)=p_{1}$, $D(0)=p_{2}, W(0)=p_{3}$ now become $L_{1}(0)=p_{1}-a, D_{1}(0)=p_{2}-b, W_{1}(0)=p_{3}-c$ and these can be written in the matrix notation as

$$
\left[\begin{array}{ccc}
L_{1}(0) & 0 & 0 \\
0 & D_{1}(0) & 0 \\
0 & 0 & W_{1}(0)
\end{array}\right]=\left[\begin{array}{ccc}
p_{1}-a & 0 & 0 \\
0 & p_{2}-b & 0 \\
0 & 0 & p_{3}-c
\end{array}\right]
$$

Now, applying Adomain's decomposition method to the system (114), we get $M X=E_{1} X B+$ $E_{2} X C+E_{1} X F_{1} X+E_{2} X F_{2} X=R X+N X$, where $R X=E_{1} X B+E_{2} X C$, the linear term and $N X=E_{1} X F_{1} X+E_{2} X F_{2} X$ is the non-linear term of the system, and $M$ denotes the differential operator. Therefore,

$$
X=M^{-1}[R X]+M^{-1}[N X]
$$

Where $X=\sum_{n=0}^{\infty} X_{n}$ and $N X=\sum_{n=0}^{\infty} A_{n}$. Thus (116) will become

$$
\sum_{n=0}^{\infty} X_{n}=M^{-1}\left\{E_{1} \sum_{n=0}^{\infty} X_{n} B+E_{2} \sum_{n=0}^{\infty} X_{n} C\right\}+M^{-1} \sum_{n=0}^{\infty} A_{n}
$$

Now the components of $X_{n}$ are therefore easily identified as,

$$
\begin{aligned}
& X_{0}=\operatorname{diag}\left[p_{1}-a, p_{2}-b, p_{3}-c\right] \\
& X_{1}=M^{-1}\left[E_{1} X_{0} B+E_{2} X_{0} C\right]+M^{-1} A_{0} \\
& X_{2}=M^{-1}\left[E_{1} X_{1} B+E_{2} X_{1} C\right]+M^{-1} A_{1} \\
& X_{n}=M^{-1}\left[E_{1} X_{n-1} B+E_{2} X_{n-1} C\right]+M^{-1} A_{n-1}
\end{aligned}
$$


Now the polynomials $A_{n}$ for the above non-linear equations are calculated by using (7) and are given by

$A_{0}=E_{1} X_{0} F_{1} X_{0}+E_{2} X_{0} F_{2} X_{0}$

$A_{1}=X_{1}\left[E_{1} F_{1} X_{0}+E_{1} X_{0} F_{1}+E_{2} F_{2} X_{0}+E_{2} X_{0} F_{2}\right]+\left(X_{1}^{2} / 2 !\right)\left[2 E_{1} F_{1}+2 E_{2} F_{2}\right]$

$A_{2}=X_{2}\left[E_{1} F_{1} X_{0}+E_{1} X_{0} F_{1}+E_{2} F_{2} X_{0}+E_{2} X_{0} F_{2}\right]+\left(X_{2}^{2} / 2 !\right)\left[2 E_{1} F_{1}+2 E_{2} F_{2}\right]+X_{1} X_{2}\left[2 E_{1} F_{1}+\right.$ $2 E_{2} F_{2}$ ].

Thus, the initial approximation matrix $X_{0}$ is given by (117) and special polynomial $A_{0}=\operatorname{diag}\left[Q_{1}, Q_{2}, Q_{3}\right]$ where $Q_{1}=-\beta_{1}\left(p_{1}-a\right)\left(p_{2}-b\right), Q_{2}=\left(p_{2}-b\right)\left[\beta_{2}\left(p_{1}-a\right)-\gamma_{2}\left(p_{3}-\right.\right.$ $c)], Q_{3}=\gamma_{3}\left(p_{2}-b\right)\left(p_{3}-c\right)$.

The second approximation $X_{1}$ is given by

$X_{1}=M^{-1}\left[E_{1} X_{0} B+E_{2} X_{0} C\right]+M^{-1} A_{0}=\operatorname{diag}\left[\left(Q_{1}+Q_{4}\right) t,\left(Q_{2}+Q_{5}\right) t,\left(Q_{3}+Q_{6}\right) t\right]$ where $Q_{4}=-a \beta_{1}\left(p_{2}-b\right), Q_{5}=b\left[\beta_{2}\left(p_{1}-a\right)-\gamma_{2}\left(p_{3}-c\right)\right], Q_{6}=c \gamma_{3}\left(p_{2}-b\right)$.

The third approximation $X_{2}$ is given by $X_{2}=M^{-1}\left[E_{1} X_{1} B+E_{2} X_{1} C\right]+M^{-1} A_{1}$.

The special polynomial $A_{1}$ is given by

$A_{1}=\operatorname{diag}\left[\begin{array}{ccc}\left(Q_{1}+Q_{4}\right) Q_{7} t & \left(Q_{2}+Q_{5}\right) Q_{8} t+ & \left(Q_{3}+Q_{6}\right) Q_{9} t+ \\ -\beta_{1}\left(Q_{1}+Q_{4}\right)^{2} t^{2} & \left(\left(\beta_{2}-\gamma_{2}\right)\left(Q_{2}+Q_{5}\right)^{2} t^{2}\right), & \gamma_{3}\left(Q_{3}+Q_{5}\right)^{2} t^{2}\end{array}\right]$ Where $Q_{7}=$ $-\beta_{1}\left(p_{1}-a+p_{2}-b\right), Q_{8}=\left(p_{2}-b\right)\left(\beta_{2}-\gamma_{2}\right)-\gamma_{2}\left(p_{3}-c\right)-\beta_{2}\left(p_{1}-a\right), Q_{9}=$ $\left.\gamma_{3}\left(p_{2}-b+p_{3}-c\right)\right)$.

Therefore $X_{2}=\operatorname{diag}\left[\left(Q_{10} t^{2}+Q_{13} t^{3}\right),\left(Q_{11} t^{2}+Q_{14} t^{3}\right),\left(Q_{12} t^{2}+Q_{15} t^{3}\right)\right]$, where $Q_{10}=\left[\left(Q_{1}+Q_{4}\right) Q_{7}-a \beta_{1}\left(Q_{2}+Q_{5}\right)\right] / 2, \quad Q_{11}=\left[\left(Q_{2}+Q_{5}\right) Q_{8}+b\left(\beta_{2}\left(Q_{1}+Q_{4}\right)-\gamma_{2}\left(Q_{3}+\right.\right.\right.$ $\left.\left.Q_{6}\right)\right] / 2, \quad Q_{12}=\left[\left(Q_{3}+Q_{6}\right) Q_{9}+\gamma_{3} c\left(Q_{2}+Q_{5}\right)\right] / 2, \quad Q_{13}=\left[-\beta_{1}\left(Q_{1}+Q_{4}\right)^{2}\right] / 3, \quad Q_{14}=$ $\left[\left(\beta_{2}-\gamma_{2}\right)\left(Q_{2}+Q_{5}\right)^{2}\right] / 3, \quad Q_{15}=\left[\gamma_{3}\left(Q_{3}+Q_{6}\right)^{2}\right] / 3$.

The fourth approximation $X_{3}$ is given by $X_{3}=M^{-1}\left[E_{1} X_{2} B+E_{2} X_{2} C\right]+M^{-1} A_{2}$. Where the special polynomial $A_{2}$ is given by $A_{2}=\operatorname{diag}\left[q_{1}, q_{2}, q_{3}\right]$, Where

$$
\begin{aligned}
q_{1}= & Q_{7} Q_{13} t^{2}+\left[Q_{7} Q_{13}-2 \beta_{2}\left(Q_{1}+Q_{4}\right) Q_{10}\right] t^{3}- \\
& {\left.\left[\beta_{2} Q_{10}^{2}+2 \beta_{2}\left(Q_{1}+Q_{4}\right) Q_{13}\right)\right] t^{4}-2 \beta_{2} Q_{10} Q_{13} t^{5}-\beta_{2} Q_{13}^{2} t^{6} } \\
q_{2}= & Q_{8} Q_{11} t^{2}+\left[Q_{8} Q_{14}+2\left(\beta_{2}-\gamma_{2}\right)\left(Q_{2}+Q_{5}\right) Q_{11}\right] t^{3}+2\left[\left(\beta_{2}-\gamma_{2}\right) Q_{11}^{2}+\left(\beta_{2}-\gamma_{2}\right)\right. \\
& \left.2\left(Q_{2}+Q_{5}\right) Q_{14}\right] t^{4}+\left(\beta_{2}-\gamma_{2}\right)\left(Q_{2}+Q_{5}\right) Q_{11} Q_{14} t^{5}+\left(\beta_{2}-\gamma_{2}\right) Q_{14}^{2} t^{6}, \\
q_{3}= & Q_{9} Q_{12} t^{2}+\left[Q_{9} Q_{15}+2 \gamma_{3}\left(Q_{3}+Q_{6}\right) Q_{12}\right] t^{3}+ \\
& {\left[\gamma_{3} Q_{12}^{2}+2 \gamma_{3} Q_{15}\left(Q_{3}+Q_{6}\right)\right] t^{4}+2 \gamma_{3} Q_{12} Q_{15} t^{5}+\gamma_{3} Q_{15}^{2} t^{6} }
\end{aligned}
$$

then the fourth approximation $X_{3}$ is calculated and is given by

$$
X_{3}=\operatorname{diag}\left[\begin{array}{ccc}
Q_{16} t^{3}+Q_{17} t^{4} & Q_{21} t^{3}+Q_{22} t^{4} & Q_{26} t^{3}+Q_{27} t^{4} \\
+Q_{18} t^{5}+Q_{19} t^{6}, & +Q_{23} t^{5}+Q_{24} t^{6},+Q_{28} t^{5}+Q_{29} t^{6} \\
+Q_{20} t^{7} & +Q_{25} t^{7} & +Q_{30} t^{7}
\end{array}\right]
$$

Where

$Q_{16}=\left[Q_{7} Q_{10}-a \beta_{1} Q_{11}\right] / 3, Q_{17}=\left[Q_{7} Q_{13}-2 \beta_{2}\left(Q_{1}+Q_{4}\right) Q_{10}-a \beta_{1} Q_{14}\right] / 4$,

$Q_{18}=\left[-\beta_{2} Q_{10}^{2}-2 \beta_{2}\left(Q_{1}+Q_{4}\right) Q_{13}\right] / 5, Q_{19}=\left[-2 \beta_{2} Q_{10} Q_{13}\right] / 6, Q_{20}=\left[-\beta_{2} Q_{13}^{2}\right] / 7, Q_{21}=$ $\left[Q_{8} Q_{14}+b \beta_{2} Q_{10}-b \gamma_{2} Q_{12}\right] / 3, Q_{22}=\left[Q_{8} Q_{14}+2\left(\beta_{2}-\gamma_{2}\right)\left(Q_{2}+Q_{5}\right) Q_{11}+b \beta_{2} Q_{13}+\right.$ $\left.b\left(\beta_{2} Q_{13}-\gamma_{2} Q_{15}\right)\right] / 4, Q_{23}=\left[\left(\beta_{2}-\gamma_{2}\right) Q_{11}^{2}+2\left(\beta_{2}-\gamma_{2}\right)\left(Q_{2}+Q_{5}\right) Q_{14}\right] / 5, Q_{24}=$ $\left[2\left(\beta_{2}-\gamma_{2}\right) Q_{11} Q_{14}\right] / 6, Q_{25}=\left[\left(\beta_{2}-\gamma_{2}\right) Q_{14}^{2}\right] / 7, Q_{26}=\left[\gamma_{3} c Q_{11}+Q_{9} Q_{12}\right] / 3, Q_{27}=$ 
$\left[Q_{9} Q_{15}+2 \gamma_{3} Q_{12}\left(Q_{3}+Q_{6}\right)+\gamma_{3} c Q_{14}\right] / 4, Q_{28}=\left[\gamma_{3} Q_{12}^{2}+2 \gamma_{3} Q_{12}\left(Q_{3}+Q_{6}\right)\right] / 5, Q_{29}=$ $\left[2 \gamma_{3} Q_{12} Q_{15}\right] / 6$ and $Q_{30}=\left[\gamma_{3} Q_{15}^{2}\right] / 7$

Thus the approximate analytical solution of (114) is given by

$$
\begin{aligned}
& X \approx X_{0}+X_{1}+X_{2}+X_{3} \text { i.e., } \\
& L_{1}=\left(p_{1}-a\right)+\left(Q_{1}+Q_{4}\right) t+Q_{10} t^{2}+\left(Q_{13}+Q_{16}\right) t^{3}+Q_{17} t^{4}+Q_{18} t^{5}+Q_{19} t^{6}+Q_{20} t^{7} \\
& D_{1}=\left(p_{2}-b\right)+\left(Q_{2}+Q_{5}\right) t+Q_{11} t^{2}+\left(Q_{14}+Q_{21}\right) t^{3}+Q_{22} t^{4}+Q_{23} t^{5}+Q_{24} t^{6}+Q_{25} t^{7} \\
& W_{1}=\left(p_{3}-c\right)+\left(Q_{3}+Q_{6}\right) t+Q_{12} t^{2}+\left(Q_{15}+Q_{26}\right) t^{3}+Q_{27} t^{4}+Q_{28} t^{5}+Q_{29} t^{6}+Q_{30} t^{7}
\end{aligned}
$$

In terms of original densities of the three species $\mathrm{L}(\mathrm{t}), \mathrm{D}(\mathrm{t})$ and $\mathrm{W}(\mathrm{t})$ the approximate solutions are given by

$$
\begin{aligned}
L(t) & =p_{1}+\left(Q_{1}+Q_{4}\right) t+Q_{10} t^{2}+\left(Q_{13}+Q_{16}\right) t^{3}+Q_{17} t^{4}+Q_{18} t^{5}+Q_{19} t^{6}+Q_{20} t^{7} \\
D(t) & =p_{2}+\left(Q_{2}+Q_{5}\right) t+Q_{11} t^{2}+\left(Q_{14}+Q_{21}\right) t^{3}+Q_{22} t^{4}+Q_{23} t^{5}+Q_{24} t^{6}+Q_{25} t^{7} \\
W(t) & =p_{3}+\left(Q_{3}+Q_{6}\right) t+Q_{12} t^{2}+\left(Q_{15}+Q_{26}\right) t^{3}+Q_{27} t^{4}+Q_{28} t^{5}+Q_{29} t^{6}+Q_{30} t^{7}
\end{aligned}
$$

The accuracy of the solution is increased by finding the higher iterations. In the absence of predators, the transformation $L=L_{1}-\alpha_{2} / \beta_{2}$ and $D=D_{1}+\alpha_{1} / \beta_{1}$ in (108) yields the following system of equations

$$
\begin{aligned}
& \dot{L}_{1}=\alpha_{1}\left(L_{1}-\alpha_{2} / \beta_{2}\right)-\beta_{1}\left(L_{1}-\alpha_{2} / \beta_{2}\right)\left(D_{1}+\alpha_{1} / \beta_{1}\right) \\
& \dot{D}_{1}=\alpha_{2}\left(D_{1}+\alpha_{1} / \beta_{1}\right)+\beta_{2}\left(L_{1}-\alpha_{2} / \beta_{2}\right)\left(D_{1}+\alpha_{1} / \beta_{1}\right) .
\end{aligned}
$$

By defining a positive definite function $V_{1}(t)$ as

$$
V_{1}(t)=d_{1}\left[\left(-\alpha_{2} / \beta_{2}\right) \log \left(1-\beta_{2} L_{1} / \alpha_{2}\right)-L_{1}\right]+d_{2}\left[\left(\alpha_{1} / \beta_{1}\right) \log \left(1+\beta_{1} D_{1} / \alpha_{1}\right)-D_{1}\right]
$$

In the absence of predators system (108) is asymptotically stable if there exists positive constants $d_{1}$ and $d_{2}$ suchthat $\left[\beta_{1} d_{1}-\beta_{2} d_{2}\right] \leq 0$ since

$$
\dot{V}_{1}(t)=-d_{1}\left[\dot{L}_{1} L_{1} /\left(L_{1}-\alpha_{2} / \beta_{2}\right)\right]-d_{2}\left[\dot{D}_{1} D_{1} /\left(D_{1}+\alpha_{1} / \beta_{1}\right)\right]=L_{1} D_{1}\left(\beta_{1} d_{1}-\beta_{2} d_{2}\right) \leq 0
$$

Similarly in the absence of litter, by defining a Liapunov function $V_{2}(t)$, a positive definite function as

$$
V_{2}(t)=d_{2}\left[\left(\alpha_{3} / \gamma_{3}\right) \log \left(1+\gamma_{3} D_{1} / \alpha_{3}\right)-D_{1}\right]+d_{3}\left[\left(\alpha_{2} / \gamma_{2}\right) \log \left(1+\gamma_{2} D_{1} / \alpha_{2}\right)-W_{1}\right]
$$

it is observed that the system (108) is asymptotically stable if there exists positive constants $d_{2}$ and $d_{3}$ suchthat $\left[\gamma_{2} d_{2}-\gamma_{3} d_{3}\right] \leq 0$. By defining a Liapunov function $V_{3}(t)$, a positive definite function as

$$
V_{3}(t)=d_{1}\left[a \log \left(1+L_{1} / a\right)-L_{1}\right]+d_{2}\left[b \log \left(1+D_{1} / b\right)-D_{1}\right]+d_{3}\left[c \log \left(1+W_{1} / c\right)-W_{1}\right]
$$

It is observed that the system (108) is asymptotically stable if there exists positive constants $d_{1}, d_{2}$ and $d_{3}$ such that $\left[\beta_{1} d_{1}-\beta_{2} d_{2}\right] \leq 0,\left[\gamma_{2} d_{2}-\gamma_{3} d_{3}\right] \leq 0$. 


\subsection{Three species system - Homotopy Analysis Method}

Consider the system of differential equations representing a three species ecological system

$$
\begin{aligned}
\dot{L}(t) & =L(t)\left[P_{1}-\gamma D(t)+\beta W(t)\right] \\
\dot{D}(t) & =D(t)\left[P_{2}+\gamma L(t)-\alpha W(t)\right] \\
\dot{W}(t) & =W(t)\left[P_{3}-\beta L(t)+\alpha D(t)\right]
\end{aligned}
$$

satisfying the initial conditions

$$
L(0)=0.2, D(0)=0.3, W(0)=0.5
$$

According to Homotopy Analysis Method, the initial approximations of (118) are satisfying the initial conditions

$$
L_{0}(0)=0.2, D_{0}(0)=0.3, W_{0}(0)=0.5
$$

and the auxiliary linear operators $\mathcal{L}$ for $i=1,2,3$ are

$$
\mathcal{L}\left[\phi_{i}(t, q)\right]=\frac{\left.\partial \phi_{i}(t, q)\right)}{\partial t}, i=1,2,3, \text { with } \mathcal{L}\left(\left[C_{i}\right]=0 .\right.
$$

and the nonlinear operators

$$
\begin{aligned}
& N_{1}\left[\phi_{i}(t, q)\right]=\frac{\left.\partial \phi_{1}(t, q)\right)}{\partial t}-\phi_{1}(t, q)\left[P_{1}-\gamma \phi_{2}(t, q)+\beta \phi_{2}(t, q)\right]=g_{1}(t) \\
& N_{2}\left[\phi_{i}(t, q)\right]=\frac{\left.\partial \phi_{2}(t, q)\right)}{\partial t}-\phi_{2}(t, q)\left[P_{2}+\gamma \phi_{1}(t, q)-\alpha \phi_{3}(t, q)\right]=g_{2}(t) \\
& N_{3}\left[\phi_{i}(t, q)\right]=\frac{\left.\partial \phi_{3}(t, q)\right)}{\partial t}-\phi_{3}(t, q)\left[P_{3}-\beta \phi_{1}(t, q)-\alpha \phi_{2}(t, q)\right]=g_{3}(t)
\end{aligned}
$$

The zeroth-order deformation equation is constructed as

$$
\begin{aligned}
(1-q) \mathcal{L}\left[\phi_{1}(t, q)-L_{0}(t)\right] & =q h\left\{N_{1}\left(\phi_{1}(t, q)\right)-g_{1}(t)\right\} \\
(1-q) \mathcal{L}\left[\phi_{2}(t, q)-D_{0}(t)\right] & =q h\left\{N_{2}\left(\phi_{2}(t, q)\right)-g_{2}(t)\right\} \\
(1-q) \mathcal{L}\left[\phi_{3}(t, q)-W_{0}(t)\right] & =q h\left\{N_{3}\left(\phi_{3}(t, q)\right)-g_{3}(t)\right\}
\end{aligned}
$$

where $q \in[0,1]$ is an embedding parameter, $h$ is a nonzero auxiliary function, $\mathcal{L}$ is an auxiliary linear operator, $L_{0}(t), D_{0}(t), W_{0}(t)$ are the initial guesses of $L(t), D(t), W(t)$, and $\phi_{i}(t, q)$ are unknown functions.

\subsubsection{Solution as polynomial functions}

Now, by taking polynomials as base functions the $m^{\text {th }}$ order deformation equations are given by

$$
\begin{aligned}
& \mathcal{L}\left[L_{m}(t)-L_{m-1}(t)\right]=q h R_{1, m}\left(\vec{L}_{m-1}\right) \\
& \mathcal{L}\left[D_{m}(t)-D_{m-1}(t)\right]=q h R_{2, m}\left(\vec{D}_{m-1}\right) \\
& \mathcal{L}\left[W_{m}(t)-W_{m-1}(t)\right]=q h R_{3, m}\left(\vec{W}_{m-1}\right)
\end{aligned}
$$


As $q$ increases from 0 to 1 , the solutions $\phi_{1}(t, q), \phi_{2}(t, q), \phi_{3}(t, q)$ vary from the initial guesses $L_{0}(t), D_{0}(t), W_{0}(t)$ to the solutions $L(t), D(t), W(t)$. where

$$
\begin{aligned}
& R_{1, m}\left(\vec{L}_{m}\right)=\dot{L}_{m-1}-P_{1} L_{m-1}(t)+\gamma \sum_{i=0}^{m-1} L_{i}(t) D_{m-1-i}(t)-\beta \sum_{i=0}^{m-1} L_{i}(t) W_{m-1-i}(t) \\
& R_{2, m}\left(\vec{L}_{m}\right)=\dot{D}_{m-1}-P_{2} D_{m-1}(t)+\gamma \sum_{i=0}^{m-1} D_{i}(t) L_{m-1-i}(t)+\alpha \sum_{i=0}^{m-1} D_{i}(t) W_{m-1-i}(t) \\
& R_{3, m}\left(\vec{W}_{m}\right)=\dot{W}_{m-1}-P_{3} W_{m-1}(t)+\beta \sum_{i=0}^{m-1} W_{i}(t) L_{m-1-i}(t)-\alpha \sum_{i=0}^{m-1} W_{i}(t) D_{m-1-i}(t) \\
& \text { subject to } L_{1, m}=0, D_{1, m}=0, W_{1, m}=0
\end{aligned}
$$

where the dot denotes differentiation with respect to the variable $t$. The solution of the $m^{\text {th }}$ order deformation equation for $m \geq 1$ is given by

$$
\begin{aligned}
& L_{m}(t)=\chi_{2} L_{m-1}(t)+h \mathcal{L}^{-1}\left[R_{1, m}\left(\vec{L}_{m-1}\right)\right] \\
& D_{m}(t)=\chi_{2} D_{m-1}(t)+h \mathcal{L}^{-1}\left[R_{2, m}\left(\vec{D}_{m-1}\right)\right] \\
& W_{m}(t)=\chi_{2} W_{m-1}(t)+h \mathcal{L}^{-1}\left[R_{3, m}\left(\vec{W}_{m-1}\right)\right] \\
& \text { and } \chi_{m}=\left\{\begin{array}{c}
0, m \leq 1 \\
1, \text { otherwise }
\end{array}\right.
\end{aligned}
$$

The integration constants $c_{i}$ are determined by the initial condition. Now the successive approximations are given by

$$
\begin{aligned}
& L_{1}(t)=\left[-0.2 P_{1} h+0.06 \gamma h-0.1 \beta h\right] t \\
& D_{1}(t)=\left[-0.3 P_{2} h-0.06 \gamma h+0.15 \alpha h\right] t \\
& W_{1}(t)=\left[-0.5 P_{3} h+0.1 \beta h-0.15 \alpha h\right] t \\
& L_{2}(t)=\left[-0.2 P_{1}+0.06 \gamma-0.1 \beta\right](h t)+\left[-0.2 P_{1}+0.06 \gamma-0.1 \beta\right]\left(h^{2} t\right)+\left[0.2 P_{1}^{2}-0.12 P_{1} \gamma+\right. \\
& \left.0.2 P_{1} \beta-0.06 P_{2} \gamma+0.006 \gamma^{2}+0.03 \alpha \gamma-0.06 \beta \gamma+0.1 P_{3} \beta+0.03 \beta^{2}+0.03 \alpha \beta\right]\left(h^{2} t^{2} / 2\right) \\
& D_{2}(t)=\left[-0.3 P_{2}-0.06 \gamma+0.15 \alpha\right](h t)+\left[-0.3 P_{2}-0.06 \gamma+0.15 \alpha\right]\left(h^{2} t\right)+\left[0.3 P_{2}^{2}+0.12 P_{2} \gamma-\right. \\
& \left.0.3 P_{2} \alpha+0.06 P_{1} \gamma-0.006 \gamma^{2}+0.03 \beta \gamma-0.06 \alpha \gamma-0.15 P_{3} \alpha+0.03 \alpha \beta+0.03 \alpha^{2}\right]\left(h^{2} t^{2} / 2\right) \\
& W_{2}(t)=\left[-0.5 P_{3}-0.1 \beta-0.15 \alpha\right](h t)+\left[-0.5 P_{3}+0.1 \beta-0.15 \alpha\right]\left(h^{2} t\right)+\left[0.5 P_{3}^{2}-0.2 P_{3} \beta+\right. \\
& \left.0.3 P_{3} \alpha-0.1 P_{1} \beta+0.03 \beta \gamma-0.03 \beta^{2}-0.06 \alpha \beta+0.15 P_{2} \alpha+0.03 \alpha \gamma-0.03 \alpha^{2}\right]\left(h^{2} t^{2} / 2\right)
\end{aligned}
$$

Now the analytic solution via the polynomial base functions of the system has the general form

$$
L(t)=\sum_{m=1}^{\infty} a_{i, m}(h) t^{m}, D(t)=\sum_{m=1}^{\infty} a_{i, m}^{\prime}(h) t^{m}, W(t)=\sum_{m=1}^{\infty} a_{i, m}^{\prime \prime}(h) t^{m}
$$

The above expressions represent a family of solution expressions in the auxiliary parameter $h$. By using h-curves [16] valid regions of a convergent solution series can be determined. Accuracy can easily be obtained by finding more terms by using symbolic computation softwares such as Maple and Mathematica. 


\subsubsection{Solution as exponential functions}

Now, by taking exponential functions as base functions, the initial approximations of (118) satisfying the initial conditions

$$
L_{0}(0)=0.2, D_{0}(0)=0.3, W_{0}(0)=0.5
$$

and the auxiliary linear operators $\mathcal{L}$ for $i=1,2,3$ are

$$
\mathcal{L}\left[\phi_{i}(t, q)\right]=\frac{\left.\partial \phi_{i}(t, q)\right)}{\partial t}+\phi_{i}(t, q), i=1,2,3, \text { with } \mathcal{L}\left(\left[C_{i} e^{-t}\right]=0 .\right.
$$

The solution of the $m^{\text {th }}$ order deformation equation for $m \geq 1$ is given by

$$
\begin{aligned}
& L_{m}(t)=\chi_{2} L_{m-1}(t)+h e^{-t} \int_{0}^{t} e^{\tau} H(\tau) R_{m}\left(\vec{L}_{m-1}\right) d \tau+C_{1} e^{-t} \\
& D_{m}(t)=\chi_{2} D_{m-1}(t)+h e^{-t} \int_{0}^{t} e^{\tau} H(\tau) R_{m}\left(\vec{D}_{m-1}\right) d \tau+C_{2} e^{-t} \\
& W_{m}(t)=\chi_{2} W_{m-1}(t)+h e^{-t} \int_{0}^{t} e^{\tau} H(\tau) R_{m}\left(\vec{W}_{m-1}\right) d \tau+C_{3} e^{-t} \\
& \text { and } \chi_{m}=\left\{\begin{array}{c}
0, m \leq 1 \\
1, \text { otherwise }
\end{array}\right.
\end{aligned}
$$

The integration constants $c_{i}$ are determined by the initial condition. Now the successive approximations obtained by taking $H(\tau)=1$ in the above equations are given by

$$
\begin{gathered}
L_{1}(t)=\left[-0.2 P_{1}+0.06 \gamma-0.1 \beta\right] h\left(1-e^{-t}\right) \\
D_{1}(t)=\left[-0.3 P_{2}-0.06 \gamma+0.15 \alpha\right] h\left(1-e^{-t}\right) \\
W_{1}(t)=\left[-0.5 P_{3}+0.1 \beta-0.15 \alpha\right] h\left(1-e^{-t}\right) \\
L_{2}(t)=\left[-0.2 P_{1}+0.06 \gamma-0.1 \beta\right] h\left(1-e^{-t}\right)+\left[-0.2 P_{1}+0.06 \gamma-0.1 \beta\right]\left(h^{2} t e^{-t}\right)+ \\
{\left[0.2 P_{1}^{2}-0.12 P_{1} \gamma+0.2 P_{1} \beta-0.06 P_{2} \gamma+0.006 \gamma^{2}+0.03 \alpha \gamma-0.06 \beta \gamma+0.1 P_{3} \beta+\right.} \\
\left.0.03 \beta^{2}+0.03 \alpha \beta\right]\left[h^{2}\left(1-e^{-t}-t e^{-t}\right)\right] \\
D_{2}(t)=\left[-0.3 P_{2}-0.06 \gamma+0.15 \alpha\right] h\left(1-e^{-t}\right)+\left[-0.3 P_{2}-0.06 \gamma+0.15 \alpha\right]\left(h^{2} t e^{-t}\right)+ \\
{\left[0.3 P_{2}^{2}+0.12 P_{2} \gamma-0.3 P_{2} \alpha+0.06 P_{1} \gamma-0.006 \gamma^{2}+0.03 \beta \gamma-0.06 \alpha \gamma-0.15 P_{3} \alpha+\right.} \\
\left.0.03 \alpha \beta+0.03 \alpha^{2}\right]\left[h^{2}\left(1-e^{-t}-t e^{-t}\right)\right] \\
W_{2}(t)=\left[-0.5 P_{3}-0.1 \beta-0.15 \alpha\right] h\left(1-e^{-t}\right)+\left[-0.5 P_{3}+0.1 \beta-0.15 \alpha\right]\left(h^{2} t e^{-t}\right)+ \\
{\left[0.5 P_{3}^{2}-0.2 P_{3} \beta+0.3 P_{3} \alpha-0.1 P_{1} \beta+0.03 \beta \gamma-0.03 \beta^{2}-0.06 \alpha \beta+0.15 P_{2} \alpha+\right.} \\
\left.0.03 \alpha \gamma-0.03 \alpha^{2}\right]\left[h^{2}\left(1-e^{-t}-t e^{-t}\right)\right]
\end{gathered}
$$

Now the analytic solution via the polynomial base functions of the system has the general form

$$
L(t)=\sum_{m=1}^{\infty} L_{m}(t), D(t)=\sum_{m=1}^{\infty} D_{m}(t), W(t)=\sum_{m=1}^{\infty} W_{m}(t)
$$

By using h-curves [16] valid regions of a convergent solution series can be determined.Accuracy can easily be obtained by finding more terms by using symbolic computation softwares such as Maple and Mathematica. 


\section{Examples}

Example 1. Consider a homogeneous system of equations

$$
\dot{x_{1}}=-102 x_{1}+88 x_{2}, \quad \dot{x_{2}}=88 x_{1}+102 x_{2}
$$

where represents derivative with respect to $t$ with the initial conditions

$$
x_{1}(0)=1, x_{2}(0)=3
$$

Now apply the Homotopy Analysis Method to solve the equations (158) by expressing the solutions $x_{1}(t)$ and $x_{2}(t)$ by a set of base functions $\left\{t^{n} / n=0,1,2 \ldots\right\}$ as $x_{1}(t)=\sum_{n=0}^{\infty} a_{n} t^{n}, x_{2}(t)=$ $\sum_{n=0}^{\infty} b_{n} t^{n}$ where $a_{n}$ and $b_{n}$ are coefficients which are to be evaluated. We choose the initial approximations as $x_{1,0}(t)=x_{1}(0)=1, x_{2,0}(t)=x_{2}(0)=3$ and the auxiliary linear operator as $L\left[\phi_{i}(t ; q)\right]=\frac{\partial \phi_{i}(t ; q)}{\partial t}, i=1,2$ with the property $L\left[C_{i}\right]=0$, where $C_{i}(i=1,2)$ are integral constants. Define a system of nonlinear operators as $N_{1}\left[\phi_{i}(t ; q)\right]=\frac{\partial \phi_{i}(t ; q)}{\partial t}+102 \phi_{1}(t ; q)-88 \phi_{2}(t ; q)$, $N_{2}\left[\phi_{i}(t ; q)\right]=\frac{\partial \phi_{i}(t ; q)}{\partial t}-88 \phi_{1}(t ; q)-102 \phi_{2}(t ; q)$. Now the zeroth order deformation equation as

$$
(1-q) L\left[\phi_{i}(t ; q)-x_{i, 0}(t)\right]=q h_{i} N_{i}\left[\phi_{i}(t ; q)\right], i=1,2
$$

From the definition of Homotopy we get $\phi_{i}(t, 0)=x_{i, 0}(t), \phi_{i}(t, 1)=x_{i}(t)$. It can be understood that as $q$ increases from 0 to 1 initial approximation $\phi_{i}(t ; q)$ approaches the solution $x_{i}(t)$ for $i=1,2$. Now $\phi_{i}(t ; q)=x_{i, 0}(t)+\sum_{m=1}^{\infty} x_{i, m}(t) q^{m}$ where

$$
x_{i, m}(t)=\left.\frac{1}{m !} \frac{\partial \phi_{i}(t ; q)}{\partial q^{m}}\right|_{q=0}
$$

Define the vector $\vec{x}_{i, n}=\left\{x_{i, 0}(t), x_{i, 1}(t), \ldots, x_{i, n}(t)\right\}$. The $m^{\text {th }}$ deformation equation is given by

$$
L\left[x_{i, m}(t)-\chi_{m} x_{i, m-1}(t)\right]=h_{i} R_{i, m} \vec{x}_{i, m}
$$

subject to the initial condition $x_{1,0}=0, x_{2,0}=0$ where

$$
\begin{aligned}
& R_{1, m} \vec{x}_{i, m}=\dot{x}_{1, m-1}+102 x_{1, m-1}-88 x_{2, m-1} \\
& R_{2, m} \vec{x}_{i, m}=\dot{x}_{2, m-1}-88 x_{1, m-1}-102 x_{2, m-1}
\end{aligned}
$$

Now the solution of the $m^{\text {th }}$ order deformation equation 162 for $m \geq 1$ is

$$
x_{i, m}(t)=\chi_{m} x_{i, m-1}(t)+h_{i} L^{-1}\left[R_{i, m}\left(\vec{x}_{i, m-1}\right)\right]
$$

Now get the successive approximations as

$$
\begin{aligned}
& x_{1,1}(t)=-120 h t, x_{2,1}(t)=280 h t, \\
& x_{1,2}(t)=-120 h t-120 h^{2} t-18400 h^{2} t^{2}, x_{2,1}(t)=2800 h t+280 h^{2} t+21600 h^{2} t^{2}
\end{aligned}
$$

Now by taking $h=-1$ (without plotting the h-curve)the solution of (158)is given by

$$
x_{1}(t)=\sum_{m=0}^{\infty} x_{1, m}(t)=-e^{-200 t}+2 e^{-40 t}, x_{2}(t)=\sum_{m=0}^{\infty} x_{2, m}(t)=e^{-200 t}+2 e^{-40 t}
$$


Example 2. Consider the system of nonlinear ordinary differential equations

$$
\dot{x_{1}}=-1002 x_{1}+1000 x_{2}^{2}, \quad \dot{x_{2}}=x_{1}-x_{2}-x_{2}^{2}
$$

with the initial conditions

$$
x_{1}(0)=1, x_{2}(0)=1
$$

Now we apply Homotopy analysis method and take the initial approximation of the solution of the above system as

$$
x_{1,0}(t)=x_{1}(0)=1, x_{2,0}(t)=x_{2}(0)=1
$$

and the auxiliary linear operator as $L\left[\phi_{i}(t ; q)\right]=\frac{\partial \phi_{i}(t ; q)}{\partial t}, i=1,2$. Define nonlinear operators as

$$
\begin{gathered}
N_{1}\left[\phi_{i}(t ; q)\right]=\frac{\partial \phi_{i}(t ; q)}{\partial t}+1002 \phi_{1}(t ; q)-1000 \phi_{2}(t ; q) \\
N_{2}\left[\phi_{i}(t ; q)\right]=\frac{\partial \phi_{i}(t ; q)}{\partial t}-\phi_{1}(t ; q)+\phi_{2}(t ; q)+\phi_{2}(t ; q)^{2}
\end{gathered}
$$

Now the zeroth order deformation equation as

$$
(1-q) L\left[\phi_{i}(t ; q)-x_{i, 0}(t)\right]=h_{i} R_{i, m}\left(\vec{x}_{i, m-1}\right) \quad i=1,2
$$

where

$$
\begin{array}{r}
R_{1, m}\left(\vec{x}_{i, m-1}\right)=\dot{x}_{1, m-1}+1002 x_{1, m-1}-1000 \sum_{j=0}^{m-1} x_{2, m-1 j}(t) \\
R_{2, m}\left(\vec{x}_{i, m-1}\right)=\dot{x}_{2, m-1}-x_{1, m-1}-\sum_{j=0}^{m-1} x_{2, m-1-j}(t)
\end{array}
$$

where the "." denotes differentiation with respect $t$. Now the solution of the $m^{\text {th }}$ order deformation is given by

$$
x_{i, m}(t)=\chi_{m} x_{i, m-1}(t)+h \int R_{i, m}\left(\vec{x}_{i, m-1}\right) d t+C_{i,} \quad i=1,2
$$

the integration constants $C i(i=1,2)$ can be determined by the initial conditions.

$$
\begin{array}{r}
x_{1,1}(t)=2 h t, x_{1,2}(t)=2 h t+2 h^{2} t+2 h^{2} t^{2}+\ldots \\
x_{2,1}(t)=h t, x_{1,2}(t)=h t+h^{2} t+h^{2} t^{2}+\ldots
\end{array}
$$

Now by taking $h=-1$ the successive approximations are computed as (The proper value of $h$ can be chosen by plotting $h$-curve of $\dot{x}_{i}(0)$ and $\dot{x}_{i}(0)$ as discussed in [16]

$$
\begin{aligned}
& x_{1,1}(t)=-2 t, x_{1,2}(t)=2 t^{2}, x_{1,3}(t)=-\frac{4}{3} t^{3} \ldots \\
& x_{2,1}(t)=-t, x_{1,2}(t)=\frac{1}{2} t^{2}, x_{1,3}(t)=-\frac{1}{6} t^{3} \ldots
\end{aligned}
$$


Thus the solution of (166) is given by

$$
\begin{gathered}
x_{1}(t)=\sum_{m=0}^{\infty} x_{1, m}(t)=\sum_{m=0}^{\infty} \frac{(-2 t)^{m}}{m !}=e^{-2 t} \\
x_{2}(t)=\sum_{m=0}^{\infty} x_{2, m}(t)=\sum_{m=0}^{\infty} \frac{(-t)^{m}}{m !}=e^{-t}
\end{gathered}
$$

The solutions (169),(170) obtained by Homotopy analysis are coinciding with the exact solutions of (166) satisfying the initial conditions (167).

Example 3. Consider the system of nonlinear ordinary differential equations

$$
\dot{x_{1}}=x_{2}, \dot{x_{2}}=x_{3}, \dot{x_{3}}=-6 x_{1}-2.92 x_{2}-x_{3}+x_{1}^{2}
$$

satisfying the initial conditions

$$
x_{1}(0)=0.2, x_{2}(0)=-0.3, x_{3}(0)=0.1
$$

Now we apply Homotopy Analysis method expressing the solutions $x_{1}(t), x_{2}(t)$ and $x_{3}(t)$ by a set of base functions $\left\{e^{(-n t)} / n \geq 0\right\}$ and take the initial approximation of the solution of the above system as

$x_{1,0}(t)=x_{1}(0)=0.2, x_{2,0}(t)=x_{2}(0)=-0.3, x_{3,0}(t)=x_{3}(0)=0.1$

and the auxiliary linear operator as $L\left[\phi_{i}(t ; q)\right]=\frac{\partial \phi_{i}(t ; q)}{\partial t}+\phi_{i}(t, q), \quad i=1,2,3$ with the property $L\left[C_{i} e^{-t}=0\right], i=1,2,3$ where $C_{i}(i=1,2,3)$ are integral constants. Now the first approximations of solutions are given by

$x_{1,1}(t)=0.3 h-0.3 h e^{-t}, x_{2,1}(t)=-0.1 h+0.1 h e^{-t}, x_{3,1}(t)=0.404 h-0.404 h e^{-t}$.

Second approximations of solutions are given by

$x_{1,2}(t)=0.3 h-0.3 h e^{-t}+0.1 h^{2}-0.1 h^{2} e^{-t}+0.2 h^{2} t e^{-t}$,

$x_{2,1}(t)=-0.1 h+0.1 h e^{-t}-0.404 h^{2}+0.404 h^{2} e^{-t}+0.304 h^{2} t e^{-t}$,

$x_{3,1}(t)=0.404 h-0.404 h e^{-t}+1.8728 h^{2}-1.8728 h^{2} e^{-t}-1.4688 h^{2} t e^{-t}$.

Now the solution of the system (171) can be expressed as $v_{i}(t)=\sum_{m=0}^{\infty} \sum_{n=0}^{\infty} d_{i, m, n}(h) t^{m} e^{-n t}, \quad i=$ $1,2,3$ where the coefficients $d_{i, m, n}$ depend on $h$ and $v_{i}(t)=x_{i}(t), i=1,2,3$. By plotting $\dot{v}(t)$ and $\ddot{v}(t)$ as discussed in [16] we can choose $h=-1$ for $x_{1}, h=-0.86$ for $x_{2}, h=-1.1$ for $x_{3}$. We can easily observe that higher order Homotopy Analysis Method solutions are very nearer to the solutions obtained by RK-method and Adams bash forth predictor corrector method.

Example 4. Consider differential equation

$$
\dot{x}=-x^{2}
$$

satisfying the initial conditions

$$
x(0)=1
$$

By applying Homotopy Perturbation method we construct Homotopy as

$$
(1-p)\left(\dot{\phi}-\dot{x}_{0}\right)+p\left(\dot{\phi}+\phi^{2}\right)=0
$$

Now the initial approximation is taken as $x_{0}=1$. Let the solution of (175) is of the form

$$
\phi=\phi_{0}+p \phi_{1}+p^{2} \phi_{2}+\ldots
$$


By equating the like powers of $p$ after using (176) in (175), we get

$\dot{\phi}_{0}=\dot{x}_{0}, \dot{\phi}_{1}+\dot{x}_{0}+\dot{\phi}_{0}^{2}=0, \phi_{1}(0)=0, \dot{\phi}_{2}+\dot{x}_{0}+2 \phi_{0} \phi_{1}=0, \phi_{2}(0)=0$. If we take $\phi_{0}=x_{0}=1$ then $\phi_{1}=-t$ and $\phi_{2}=t^{2}$. Thus the second order approximation of the solution of (175) obtained by using Homotopy Perturbation method is given by $x(t)=\phi_{0}+p \phi_{1}+p^{2} \phi_{2}=1-t+t^{2}$.

Example 5. Consider the system of nonlinear ordinary differential equations

$$
\dot{x_{1}}=x_{1}-x_{1} x_{2}, \dot{x_{2}}=-0.1 x_{2}+x_{1} x_{2}
$$

with the initial conditions $x_{1}(0)=14, x_{2}(0)=18$. By applying Homotopy perturbation method we get the third order approximation to the solution as $x_{1}=14-238 t+271.6 t^{2}+20191.32 t^{3}$,

$x_{2}=18+250.2 t-403.11 t^{2}-20087.33 t^{3}$. We can observe that higher order approximations agree with the solutins obtained by RK-method and Adams bash forth predictor corrector method.

\section{Summary and conclusions}

In this chapter we studied the dynamics of Two species and Three species ecological systems. Since Two species systems represent the various prey predator interactions and Three species systems represent the behavior of the litter, detritus and predators in the mangrove areas their respective dynamics are modeled by nonlinear differential equations. Heuristic procedures for Perturbation method, Adomian decomposition method for system, Homotopy perturbation method and Homotopy analysis method are presented so as to familiarize the reader with the application of these important methods. Two species and Three species models are solved by using the above stated methods. The Homotopy perturbation method which was used to solve the nonlinear system of differential equations, governing the prey and predator problem, is very easy and accurate to employ with reliable results. There is less computation needed in comparison with the Adomian decomposition and power series methods.

Homotopy analysis method was applied to solve both the Two species and Three species ecological systems by considering both Polynomial base functions and exponential base functions. The accuracy of the continuous solution obtained by using Homotopy analysis method ia almost same as that of a numerical method. This is convenient for practical applications with minimum requirements on calculation and computation and validity of the Homotopy analysis method series solutions can be enhanced by finding more terms and/or using the Pade technique. The functional form of the solution would be useful in the study of the stability of the system. Owing to the generality, the considered two species and Three species models exhibit very rich dynamics. From the illustrative examples it can be observed that Homotopy analysis method is a powerful method for nonlinear problems and provides us with a convenient way of controlling the convergence of approximation series, which is a major advantage when compared with other methods.

\section{Acknowledgements}

This work is supported by DST-CMS project Lr.No.SR/S4/MS:516/07, Dt.21-04-2008 and the support is gratefully acknowledged.

\section{References}

[1] Pielou, E.C, An Introduction to Mathematical Ecology, Wiley-Interscience, New York (1969) 
[2] Rao C.R and Mitra S.K, Generalized Inverse of Matrices and its Applications, John Wiley and Sons, New York (1971)

[3] Murty K.N and Rao D.V.G, Approximate analytical solution of general Lotka-Volterra equations, J. Math. Anal. Appl. 122(1987), pp. 582-588

[4] Murty K.N, Srinivas M.A.S and Prasad K.R, Certain mathematical models for biological systems and their approximate analytical solutions, In: Proc. Int. Conf. on Non-linear analysis and applications to Bio-mathematics, Andhra Univ., Visakhapatnam, 1987

[5] Adomian G, A review of the decomposition method in applied mathematics, J. Math. Anal. App. 135(1988), pp. 501-544

[6] Venkata Sundaranand Putcha, Ramu Malladi., A Mathematical Model on Detritus In Mangrove Estuarine Ecosystem, International Journal of Pure and Applied Mathematics Volume 63 No. 2 2010, 169-181

[7] Murty K.N, Srinivas M.A.S and Prasad K.R, Approximate analytical solutions to the three-species ecological system, J. Math. Anal. Appl. 145(1990), pp. 89-99

[8] Nayfeh A.H, Perturbation Methods, Wiley Classics Library, John Wiley \& Sons, New York, NY, USA, 2000.

[9] Srinivasu P.D.N and Gayatri I.L, Influence of prey reserve capacity on predator-prey dynamics, Ecological Modelling 181(2005), pp. 191-202

[10] Murray J. D, Mathematical Biology I An Introduction, 3rd ed, Springer, Berlin, 2002

[11] Freedman H.I and Waltman P, Persistence in models of three interacting predator-prey populations, Mathematical Biosciences 68(1984), No. 2, 213Ü231

[12] He J.H, Homotopy perturbation technique, Computer Methods in Applied Mechanics and Engineering 178 (3Ü4) (1999) 257Ü262.

[13] He J.H, Homotopy perturbation technique:A new nonlinear analytical technique, Applied Mathematics and Computation 135(2003) 73Ü79.

[14] He J.H, Asymptotology by Homotopy perturbation method, Applied Mathematics and Computation 156(2004) 591Ü596.

[15] Liao S.J., The proposed Homotopy analysis techniques for the solution of nonlinear problems, Ph.D. dissertation, Shanghai Jiao Tong University, Shanghai, China, 1992.

[16] Liao S.J, Beyond perturbation, Introduction to the Homotopy analysis method, CRC Press, Boca Raton, Chapman and Hall, 2003

[17] Varma V.S, Exact Solutions for a special Prey Predator Competing species system, Bulltin Mathematical Biology, 39 (1977) 619-622

[18] Wilson, A J, On Varma's Prey Predaor Poblem, 42 (1980) 599-600

[19] Burnside R.R, A note on exact Solutions of Prey Predator equations, Bulltin Mathematical Biology, 44 (1982) 893-897

[20] Lotka A.J, Elements of Physical Biology, Williams \& Wilkins, Baltimore, Md, USA, 1925.

[21] Ayala F.J, Gilpin M.E and Ehrenfeld J.G, Competition between species: Theoretical Models and experimental tests, Theoret. Population Biology,4 (1973), 331-356

[22] Albrecht F, Gatzke H, Haddad A and Wax N, The dynamics of two interacting Populations, Journal of Mathematical Analysis and Applications, 46 (1974), 658-670

[23] Gopalaswamy K, Time lags and global Stability in two species competition, Bulletin Mathematical Biology,42 (1980), 728-737

[24] Brown G.C, Stability in an insect pathogen model incorporating age-dependent immunity and seasonal host reproduction, Bulletin Mathematical Biology,46 (1984), 139-153 
[25] May R.M and Leonard W.J, Nonlinear aspects of competition between three species, SIAM Journal of Applied Mathematics,29,2 (1975), 243-253

[26] Schuster P , Sigmund K and Wolff R, on w-limits for competition between three species, SIAM Journal of Applied Mathematics, 29,1 (1979), 49-54

[27] Zhangjinyan, Three dimensional Gradiant conjugate system and applications, Proceedings of the International Conference on Non-linear analysis and applications, USA, 109 (1986) 613-621.

[28] Venkata Sundaranand Putcha and Ramu Malladi, A Mathematical Model on Detritus In Mangrove Estuarine Ecosystem, International Journal of Pure and Applied Mathematics, 63, 2 (2010), 169-181

[29] Venkata Sundaranand Putcha, Non-Linear Differential and Difference Equations Existence, Uniqueness, Stability, Observability and Controllability, Ph.D Dissertation, Andhra University, Visakhapatnam, India, 1995

[30] Mantripragada Ananta Satya Srinivas, Contributions to Certain Nonlinear Biomathematical Models, Ph.D Thesis, Andhra University, Visakhapatnam, India, 1989

[31] John Pastor, Mathematical Ecology of Populations and Ecosystems, Wiley-Blackwell , A John Wiley \& Sons, Ltd., Publication, 2008

[32] Sami Bataineh A, Noorani M.S.M, Hashim I, Solving systems of ODEs by Homotopy analysis method, Communications in Nonlinear Science and Numerical Simulation 13 (2008) 2060-2070

[33] Francisco M. Fernt'andez, On some approximate methods for nonlinear Models, arXiv:0904.4044v1 [math-ph] 26 Apr 2009

[34] Faghidian Sa, Moghimi Zand, M, Farjami Y, Farrahi G.H, Application of Homotopy-Pade Technique To The Volterra's Prey And Predator Problem, Appl. Comput. Math., V.10, N.2, 2011, pp.262-270

[35] Shijun Liao, Comparison between the Homotopy analysis method and Homotopy perturbation method, Applied Mathematics and Computation 169 (2005) 1186-1194

[36] Zoua L, Zonga Z, Dongb G.H, Generalizing Homotopy analysis method to solve Lotka-Volterra equationI, Computers and Mathematics with Applications 56 (2008) 2289-2293

[37] Rafei M, Daniali H, Ganji D.D, Pashaei H, Solution of the prey and predator problem by Homotopy perturbation method, Applied Mathematics and Computation 188 (2007) 1419-1425

[38] Cheng-shi Liu, The essence of the Homotopy analysis method, arXiv:1105.6183v1 [nlin.SI] 31 May 2011

[39] Vipul K Baranwal, Ram K Pandey, Manoj P Tripathi, Om P Singh, Analytic algorithms for Some Models of Nonlinear Age-Structured Population Dynamics and Epidemiology, Journal of Modern Physics, 2011, 2, 236-247 


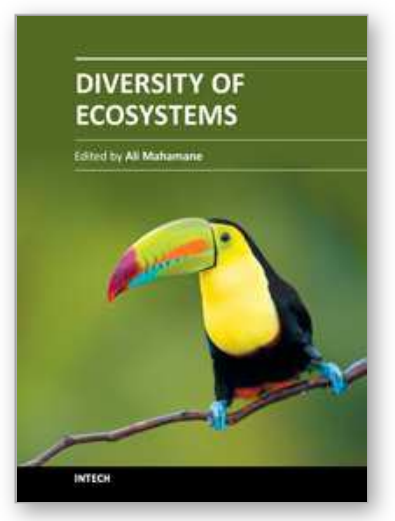

\author{
Diversity of Ecosystems \\ Edited by Prof. Mahamane Ali
}

ISBN 978-953-51-0572-5

Hard cover, 484 pages

Publisher InTech

Published online 27, April, 2012

Published in print edition April, 2012

The ecosystems present a great diversity worldwide and use various functionalities according to ecologic regions. In this new context of variability and climatic changes, these ecosystems undergo notable modifications amplified by domestic uses of which it was subjected to. Indeed the ecosystems render diverse services to humanity from their composition and structure but the tolerable levels are unknown. The preservation of these ecosystemic services needs a clear understanding of their complexity. The role of research is not only to characterise the ecosystems but also to clearly define the tolerable usage levels. Their characterisation proves to be important not only for the local populations that use it but also for the conservation of biodiversity. Hence, the measurement, management and protection of ecosystems need innovative and diverse methods. For all these reasons, the aim of this book is to bring out a general view on the function of ecosystems, modelling, sampling strategies, invading species, the response of organisms to modifications, the carbon dynamics, the mathematical models and theories that can be applied in diverse conditions.

\title{
How to reference
}

In order to correctly reference this scholarly work, feel free to copy and paste the following:

Venkata Sundaranand Putcha (2012). Two Species and Three Species Ecological Modeling - Homotopy Analysis, Diversity of Ecosystems, Prof. Mahamane Ali (Ed.), ISBN: 978-953-51-0572-5, InTech, Available from: http://www.intechopen.com/books/diversity-of-ecosystems/two-species-and-three-species-ecologicalmodeling-homotopy-analysis

\section{INTECH}

open science | open minds

\section{InTech Europe}

University Campus STeP Ri Slavka Krautzeka 83/A

51000 Rijeka, Croatia

Phone: +385 (51) 770447

Fax: +385 (51) 686166

www.intechopen.com

\section{InTech China}

Unit 405, Office Block, Hotel Equatorial Shanghai

No.65, Yan An Road (West), Shanghai, 200040, China 中国上海市延安西路65号上海国际贵都大饭店办公楼 405 单元 Phone: +86-21-62489820

Fax: +86-21-62489821 
(C) 2012 The Author(s). Licensee IntechOpen. This is an open access article distributed under the terms of the Creative Commons Attribution 3.0 License, which permits unrestricted use, distribution, and reproduction in any medium, provided the original work is properly cited. 